\title{
VOLTERRA SERIES AND PERMUTATION GROUPS
}

\author{
A. A. Agrachev and R. V. Gamkrelidze
}

UDC 512.542.7+517.972.1

Algebraic structures, connected with the asymptotic expansions of perturbations of smooth dynamical systems, are investigated; first of all, the so-called shuffle multiplication for permutations and for iterated integrals.

\section{INTRODUCTION. VARIATIONS OF A DYNAMICAL SYSTEM}

1. We consider a system of differential equations

$$
x=i_{1}(x), x \in M
$$

on a manifold $M$ of class $C^{\infty}$ with a fixed initial condition $x(0)=x_{0}$ and then we perturb this system by adding to the righthand side a vector field $\varepsilon g_{l}(x)$, where $\varepsilon$ is a small parameter. We obtain the system

$$
\dot{x}=f_{t}(x)+\varepsilon g_{t}(x), x(0)=x_{0}
$$

Let $\mathrm{x}_{\varepsilon}(\mathrm{t}), 0 \leq \mathrm{t} \leq 1$, be the solution of the system (1.1). We pose the classical question: how to find the tangent to the curve $\varepsilon \rightarrow \mathrm{x}_{\varepsilon}(1)$ for $\varepsilon=0$. The answer can be found in any manual on ordinary differential equations: one has to solve the linear system of equations in variations

$$
\dot{\xi}==\left.\frac{\partial f_{t}}{\partial x}\right|_{x_{0}(t)} \xi+g_{t}\left(x_{0}(t)\right), \quad \xi(0)=0
$$

Then $\xi(1)$ is the desired tangent vector in the case when $\xi(1) \neq 0$. However, we are concerned with those more interesting cases when $\xi(1)=0$. In this case one has to consider higher terms in the Taylor expansion of the curve $\varepsilon \rightarrow \mathrm{x}_{\varepsilon}(1)$ (higher variations of the system (1)). However, as it is known, the higher terms of the Taylor expansion do not have an invariant meaning: they are correctly defined only in fixed local coordinates. Under nonlinear changes of coordinates, the various terms of the series are intermixed and may appear and disappear depending on the choice of the coordinates. Indeed, it is obvious that there exist always local coordinates in which a segment of the given smooth curve for $\varepsilon \geq 0$ can be represented by a segment of a straight line.

A given n-th term of the Taylor expansion is a correctly defined tangent vector only in the case when all the terms with smaller index are equal to zero.

Thus, we must draw the following conclusion: the $\mathrm{n}$-th variation of the system (1.1) is what is left from the $\mathrm{n}$-th term of the Taylor expansion of the curve $\varepsilon \rightarrow \mathrm{x}_{\varepsilon}(1)$ in the case when all the preceding terms are equal to zero. Naturally, if the initial point $x_{0}$ and the fields $f_{t}, g_{t}$ are given, then the computation of the first nonzero term of the Taylor expansion is not a problem. However, with the variation of the initial data, the index of the first nonzero term changes. At the same time it is important in the theory to have the higher variations as some universal forms, into which one can substitute the initial data without knowing beforehand how many terms of the expansion are equal to zero.

Translated from Itogi Nauki i Tekhniki, Seriya Sovremennye Problemy Matematiki, Noveishie Dostizheniya, Vol. 39, pp. 3-40, 1991. 
In other words, the $\mathrm{n}$-th variation is that new object that contains the $\mathrm{n}$-th term of the expansion in comparison with the previous terms. One of the problems of this paper is to separate this new object so that the higher variations be finally describable in terms of concrete mathematical structures. The subsequent investigation is determined by the character of these structures.

2. In order to move forwards we need some notations and formulas from chronological calculus. They are described briefly in this subsection. For details see [1], [2]. Chronological calculus is a variant of operational calculus, enabling us, at least for formal calculations, to work with nonlinear dynamical systems like with linear ones.

Let $C^{\infty}(M)$ be the algebra of all real infinitely differentiable functions on $M$. We identify an arbitrary point $x \in M$ with the homomorphism $\varphi \rightarrow \varphi(x), \varphi \in C^{\infty}(M)$, of the algebra $C^{\infty}(M)$ into $\mathbf{R}$; we identify a diffeomorphism $\mathbf{p}: M \rightarrow M$ with the automorphism of the algebra $C^{\infty}(M)$, obtained if to an arbitrary function on $M$ one associates the superposition of this function and $p$ :

$$
\varphi(\cdot) \mapsto \varphi(p(\cdot)) \quad \forall \varphi \in C^{\infty}(M)
$$

The value of the diffeomorphism $\mathrm{p}$ at the point $\mathrm{x}$ is denoted by $\mathrm{x} \circ \mathrm{p}$, i. e. the composition of the automorphism of the algebra $C^{\infty}(M)$ and the homomorphism of this algebra into $\mathbf{R}$ (which again is a homomorphism of $C^{\infty}(M)$ into $\mathbf{R}$.

The smooth vector fields on $M$ are identified with the derivations of the algebra $C^{\infty}(M)$, i. e. $R$-linear mappings $f$ : $\mathrm{C}^{\infty}(\mathrm{M}) \rightarrow \mathrm{C}^{\infty}(\mathrm{M})$, satisfying the Leibnitz rule: $\mathrm{f}\left(\varphi_{1} \varphi_{2}\right)=\left(\mathrm{f} \varphi_{1}\right) \varphi_{2}+\varphi_{1}\left(\mathrm{f} \varphi_{2}\right)$. The Lie bracket $[\mathrm{f}, \mathrm{g}]=\mathrm{f} \circ \mathrm{g}-\mathrm{g} \circ \mathrm{f}$ defines a Lie algebra structure on the space $\operatorname{Vect}(\mathrm{M})$ of all smooth vector fields. In local coordinates $x=\left(x_{1}, \ldots, x_{11}\right)$, in which the vector fields have the form

$$
f=\sum_{i=1}^{m} f^{l} \frac{\partial}{\partial x_{i}}, \quad g=\sum_{i=1}^{\prime \prime} g^{i} \frac{\partial}{\partial x_{i}},
$$

the Lie brackets are computed in the following manner:

$$
[f, g]=\sum_{i=1}^{m} \sum_{j=1}^{m}\left(\frac{\partial g^{i}}{\partial x_{j}} f_{j}-\frac{\partial f^{l}}{\partial x_{j}} g_{j}\right) \frac{\partial}{\partial x_{i}} .
$$

The tangent vectors $\xi \in \mathrm{T}_{\mathbf{x}} \mathrm{M}$ are $\mathbf{R}$-linear mappings $\xi: \mathrm{C}^{\infty}(\mathrm{M}) \rightarrow \mathbf{R}$, satisfying the condition: $\xi\left(\varphi_{1} \varphi_{2}\right)=\left(\xi \varphi_{1}\right) \varphi_{2}(\mathrm{x})$ $+\varphi_{1}(x)\left(\xi \varphi_{2}\right)$. The value of the field $f$ at the point $x$ is denoted by $x \circ f$, i.e., the composition of the operator $f$ and the functional $\mathrm{x}$, which is the tangent vector at the point $\mathrm{x}$.

Each diffeomorphism $p$ (considered as an automorphism of the algebra $\mathrm{C}^{\infty}(\mathrm{M})$ ) defines the adjoint automorphism Ad $p$ of the Lie algebra Vect(M), acting according to the rule (Ad $p) f=p \circ f \circ p^{-1}$. We note that $A d p^{-1}$ is nothing else but the differential of $\mathrm{p}$; in more traditional notations: $\mathrm{p}_{*}=A d \mathrm{p}^{-1}$.

Finally, for each $f \in \operatorname{Vect}(M)$ by ad $f: \operatorname{Vect}(M) \rightarrow \operatorname{Vect}(M)$ one denotes, as usually, the operation of left Lie multiplication: $(\mathrm{ad}) \mathrm{g}=[\mathrm{f}, \mathrm{g}]$.

In the described system of notations, a diffeomorphism, denoted by the same symbol p, may be considered both a smooth transformation of the manifold and a linear operator on $C^{\infty}(M)$. This, however, cannot lead to ambiguity since in each formula the meaning of the symbol is uniquely recovered from the fact whether the point of the manifold is placed to the right or to the left of $p$.

The algebra $\mathrm{C}^{\infty}(\mathrm{M})$ is considered with the standard topology of the convergence of all the derivatives on compacta. This topology is defined by the system of seminorms $\|\cdot\|_{N, K}, N \geq 0, K \Subset M$, and the convergence $\left\|\varphi_{n}\right\|_{N, K} \rightarrow 0(n \rightarrow \infty)$ means that all the derivatives up to order $\mathrm{N}$ of the function $\varphi$ tend to zero, uniformly on $\mathrm{K}$. The seminorms $\|\cdot\|_{\mathrm{N}, \mathrm{K}}$, unlike the topology induced by them, are not uniquely defined and can be selected in various manners. However, we shall assume that the selection has been realized and the seminorms are fixed once and for all.

For a vector field $f \in \operatorname{Vect}(M)$ we define the seminorms

$$
\|f\|_{N, K}=\sup \left\{\|f \varphi\|_{N, K} \mid\|\varphi\|_{N+1, K}=1\right\} .
$$

We will have to deal constantly with one-parameter families of linear operators and functionals on $\mathrm{C}^{\infty}(\mathrm{M})$ or on $\operatorname{Vect}(\mathrm{M})$ 
The concepts of continuity, summability, differentiability, absolute continuity, etc. are defined "pointwise": we say that a family $A_{t}: C^{\infty}(M) \rightarrow C^{\infty}(M)$ of linear operators (or $A_{t}: C^{\infty}(M) \rightarrow R$ of linear functionals) has one of the enumerated properties if the families $t \rightarrow A_{t} \varphi$ have this property for each $\varphi \in C^{\infty}(M)$ (for details see [1], [2]). The same goes for families of operators and functionals on Vect(M).

Families $f_{t} \in \operatorname{Vect}(M)$, locally integrable with respect to $t \in \mathbf{R}$, are called nonstationary vector fields on $M$, while families of diffeomorphisms $p_{t} \in \operatorname{Diff(M)}$, absolutely continuous with respect to $t$, are called (nonstationary) flows on $M$.

A nonstationary vector field $f_{t}, t \in R$, defines the ordinary differential equation $(d x / d t)(t)=x(t) \circ f_{t}, x \in M$.

A field $f_{t}$ is said to be complete if all the solutions of this equation are defined for all $t \in \mathbf{R}$. Each complete field $f_{t}$ generates a flow $p_{t}, t \in \mathbf{R}$, which is the unique absolutely continuous solution of the operator equation

$$
\frac{d}{d t} p_{t}=p_{t} \circ f_{t}, \quad p_{0}=\mathrm{id}
$$

where id is the identity operator (the identity diffeomorphism of $M$ ). We call this flow the right chronological exponent of $f_{\mathfrak{t}}$ and we denote it $\mathrm{p}_{\mathrm{t}}=\mathrm{e} \overrightarrow{\mathrm{xp}} \int_{0} \mathrm{t}_{\tau} \mathrm{d} \tau$. One has an asymptotic representation of the chronological exponent in the form of a so-called Volterra series:

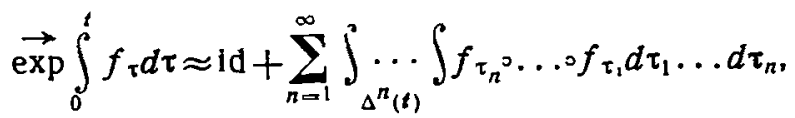

where $\Delta^{\mathrm{n}}(\mathrm{t})=\left\{\left(\tau_{1}, \ldots, \tau_{\mathrm{n}}\right) \mid 0 \leq \tau_{\mathrm{n}} \leq \ldots \leq \tau_{1} \leq \mathrm{t}\right\}$ (for $\mathrm{t}=1$ we shall use the abbreviated notation $\Delta^{\mathrm{n}}(1)=\Delta^{\mathrm{n}}$ ).

The remainder in the asymptotic expansion (1.3) is estimated in the following manner:

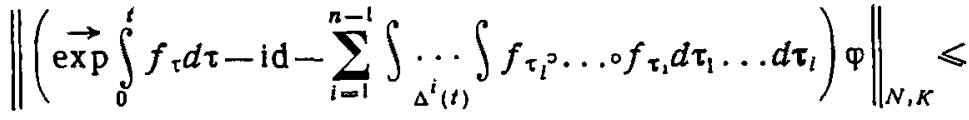

$$
\begin{aligned}
& \leqslant c_{1} e^{c \int_{0}^{t}\left\|f_{\tau}\right\|_{N, K^{\prime} d \tau}}\left(\int_{0}^{t}\left\|f_{\tau}\right\|_{N+n-1, K^{\prime}} d \tau\right)^{n}\|\varphi\|_{n+N, K^{\prime}},
\end{aligned}
$$

where $K^{\prime}$ is some (compact) neighborhood of the compactum $K$, while $c_{1}$, $c_{2}$ depend neither on $f_{\tau}$ nor on $\varphi \in C^{\infty}(M)$.

If $p_{t}=\overrightarrow{\exp } \int_{0}^{t} f_{\tau} d \tau$, then $\mathrm{p}_{\mathrm{t}}^{-1}$ satisfies the equation

$$
\frac{d}{d t} p_{t}^{-1}=-f_{t}, p_{t}^{-1}, \quad p_{0}^{-1}=\mathrm{id}
$$

Moreover, the flow $\mathrm{P}_{\mathrm{t}}^{-1}, \mathrm{t} \in \mathbf{R}$, is the unique solution, absolutely continuous with respect to $t$, of the equation $(d / d t) Q_{t}=$ $-f_{t} \circ Q_{t}$ with initial condition $Q_{0}=i d$. We call this flow the left chronological exponent of $\left(-f_{t}\right)$ and we denote it $p_{t}{ }^{-1}=$ $\exp \int_{0}^{t}-f_{\tau} d \tau$. We have the asymptotic expansion

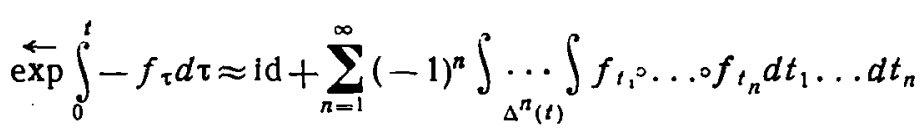

with an estimate of the remainder similar to (1.4).

Further, the family of operators $A d p_{t}: \operatorname{Vect}(M) \rightarrow \operatorname{Vect}(M), t \in R$, satisfies the equation $(d / d t) A d p_{t}=A d p_{t} \circ a d f_{t}$. Indeed,

$$
\frac{d}{d t}\left(\operatorname{Ad} p_{t}\right) g=\frac{d}{d t}\left(p_{t} \circ g^{\circ} p_{t}^{-1}\right)=p_{t} \circ\left(f_{t} \circ g-g_{\circ} f_{t}\right) \circ p_{t}^{-1}=\operatorname{Ad} p_{t}\left[f_{t}, g\right]
$$

Moreover, the family Ad $\mathrm{p}_{t}$ is the unique solution of the operator equation $\frac{d}{d t} \mathscr{P}_{t}=\mathscr{P}_{t}$ ad $f_{t}$ with initial condition $\mathscr{P}_{0}=$ Id and, therefore, the following notation is natural:

$$
\operatorname{Ad}\left(\overrightarrow{\exp } \int_{0}^{t} f_{\tau} d \tau\right)=\overrightarrow{\exp } \int_{0}^{t} \operatorname{ad} f_{\tau} d \tau
$$


We have the asymptotic expansion

$$
\operatorname{Ad}\left(\exp \int_{0}^{i} f_{\tau} d \tau\right) \approx \mathrm{Id}+\sum_{n=1}^{\infty} \int \underset{\Delta^{n}(t)}{\int} \ldots \int \operatorname{ad} f_{\tau_{n} \circ} \ldots \text { odd } f_{\tau} d \tau_{1} \ldots d \tau_{n}
$$

with an estimate of the remainder similar to (1.4). For a stationary field $\mathrm{f}_{\mathrm{t}} \equiv \mathrm{f}$ we make use of the traditional notation

$$
\overrightarrow{\exp } \int_{0}^{t} f d \tau=\exp \int_{0}^{t} f d \tau=e^{t \prime}
$$

Let $f_{t}, f_{t}+g_{t}$ be complete nonstationary fields. We have the following "variation formula" (which is a straightforward generalization of Lagrange's variation of constant formula):

$$
\overrightarrow{\exp } \int_{0}^{t}\left(f_{\tau}+g_{\tau}\right) d \tau=\overrightarrow{\exp } \int_{0}^{t}\left(\overrightarrow{\exp } \int_{0}^{\tau} \operatorname{ad} f_{\theta} d \theta g_{\tau}\right) d \tau \overrightarrow{\exp } \int_{0}^{t} f_{\tau} d \tau
$$

Indeed, we set

$$
C_{t}=\overrightarrow{\exp } \int_{0}^{t} f_{\tau}+g_{\tau} d \tau\left(\overrightarrow{\exp } \int_{0}^{t} f_{\tau} d \tau\right)^{-1}=\overrightarrow{\exp } \int_{0}^{t} f_{\tau}+g_{\tau} d \tau \circ \leftarrow \exp \int_{0}^{t}-f_{\tau} d \tau
$$

Then

$$
\begin{gathered}
\frac{d}{d t} C_{t}=\exp \int_{0}^{t}\left(f_{\tau}+g_{\tau}\right) d \tau \circ g_{t} \stackrel{\leftarrow}{\leftarrow p} \int_{0}^{t}-f_{\tau} d \tau=C_{t}=\operatorname{Ad}\left(\overrightarrow{\exp } \int_{0}^{t} f_{\tau} d \tau\right) g_{t}= \\
=C_{t} \exp \int_{0}^{t} \operatorname{ad} f_{\tau} d \tau g_{t}, \quad C_{0}=\mathrm{id} .
\end{gathered}
$$

Consequently, $C_{t}=\overrightarrow{\exp } \int_{0}^{t}\left(\overrightarrow{\exp } \int_{0}^{\tau} \operatorname{ad} f_{n} d \theta g_{\tau}\right) d \tau$. Making use of the variation formula, it is easy to find the directional derivatives of the mapping e $\bar{x}$, associating to a nonstationary field a flow on M. Namely, let $p_{t}(\varepsilon)=\exp \int_{0}^{t}\left(f_{\tau}+\varepsilon g_{\tau}\right) d \tau, \varepsilon \in \mathbf{R}$. Then

$$
p_{t}\left(\varepsilon+\varepsilon^{\prime}\right)=\overrightarrow{\exp } \int_{0}^{t} \varepsilon^{\prime}\left(\operatorname{Ad} p_{\tau}(\varepsilon)\right) g_{\tau} d \tau p_{t}(t)=\left(\mathrm{id}+\varepsilon^{\prime} \int_{0}^{t}\left(\operatorname{Ad} p_{\tau}(\varepsilon)\right) g_{\tau} d \tau+\ldots\right) \circ p_{t}(\varepsilon)
$$

Correspondingly,

$$
\begin{aligned}
& \frac{\partial}{\partial \varepsilon} p_{t}(\varepsilon)=\int_{0}^{t}\left(\operatorname{Ad} p_{\tau}(\varepsilon)\right) g_{\tau} d \tau \circ p_{t}(\varepsilon)=\int_{0}^{t} \overrightarrow{\exp } \int_{0}^{\tau} \operatorname{ad}\left(f_{\theta}+\varepsilon g_{\theta}\right) d \theta g_{\tau} d \tau \circ p_{t}(\varepsilon)= \\
& =p_{t}(\varepsilon) \circ \int_{0}^{t}\left(\operatorname{Ad} p_{t}^{-1}(\varepsilon) \circ p_{\tau}(\varepsilon)\right) g_{\tau} d \tau=p_{t}(\varepsilon) \circ \int_{0}^{t} \exp \int_{\tau}^{t}-\operatorname{ad}\left(f_{\theta}+\varepsilon g_{\theta}\right) d \theta g_{\tau} d \tau .
\end{aligned}
$$

3. We return to the system (1.1). In accordance with the variation formula we obtain

$$
x_{\varepsilon}(1)=x_{0} \circ \overrightarrow{e x p} \int_{0}^{1}\left(f_{t}+\varepsilon g_{t}\right) d t=x_{0} \circ \overrightarrow{e x p} \int_{0}^{1} \varepsilon h_{t} d t \circ \overrightarrow{e x p} \int_{0}^{1} f_{t} d t,
$$

where

$$
h_{t}=\left(\operatorname{Ad} \overrightarrow{\exp } \int_{0}^{t} f_{\tau} d \tau\right) g_{t}=\overrightarrow{\exp } \int_{0}^{t} \operatorname{ad} f_{\tau} d \tau g_{t}
$$


If we set $q(\varepsilon)=x_{0} \circ \overrightarrow{e x p} \int_{0}^{t} \varepsilon h_{t} d t$, then $x_{\varepsilon}(1)=q(\varepsilon) \circ \overrightarrow{\exp } \int_{0}^{1} f_{t} d t$.

Since the diffeomorphism $\overrightarrow{\exp } \int_{0}^{1} f_{t} d t$ does not depend on $\varepsilon$, we have the right not to turn our attention to it and instead of the curve $x_{\varepsilon}(1)$ to investigate the curve $q(\varepsilon)$, corresponding to the system

$$
\dot{x}=\varepsilon x h_{i}, \quad x(0)=x_{0},
$$

homogeneous with respect to $\varepsilon$. We have

$$
q(\varepsilon)=x_{0^{\circ}}\left(1 \mathrm{~d}+\varepsilon \int_{0}^{1} h_{t} d t+\varepsilon^{2} \iint_{\Delta^{2}} h_{t,}, h_{t_{1}} d t_{1} d t_{2}+\ldots\right)
$$

Consequently, the first variation of the system (1.8) is the tangent vector $x_{0} \int_{0}^{1} h_{t} d t$.

The coefficient of $\varepsilon^{2}$ in the expansion (1.9) is the composition of $x_{0}$ with a second-order differential operator, i.e., it is in no way a tangent vector. However, according to the general rule, this coefficient must coincide with some tangent vector at the point $x_{0}$ in the case when $x_{0} \int_{0}^{l} h_{t} d t=0$. It is easy to find the explicit expression of this tangent vector in terms of $h_{t}$. Indeed,

$$
\begin{gathered}
\iint_{\Delta^{2}} h_{t_{2}} \circ h_{t_{1}} d t_{1} d t_{2}=\iint_{\Delta^{2}}\left(h_{t_{1}} \circ h_{t_{1}}+h_{t_{1}} \circ h_{t_{3}}\right) d t_{1} d t_{2}+ \\
+\iint_{\Delta^{2}}\left[h_{t_{3}}, h_{t_{1}}\right] d t_{1} d t_{2}=\left(\int_{0}^{1} h_{t} d t\right) \circ\left(\int_{0}^{1} h_{t} d t\right)+\int_{\Delta^{2}} \int_{0}\left[h_{t_{1},}, h_{t_{1}}\right] d t_{1} d t_{2} .
\end{gathered}
$$

The commutator $\left[h_{t_{2}}, h_{t_{1}}\right]$ of vector fields is again a vector field. Consequently, the desired tangent vector has the form

$$
\frac{1}{2} x^{0} 0 \int_{\Delta^{2}}\left[h_{t_{2}}, h_{t_{1}}\right] d t_{1} d t_{2}
$$

We note that the expression (1.10), i.e., the second variation of the system (1.8), is a correctly defined tangent vector, independent of the fact whether the first variation is equal to zero.

It is natural to assume that in any case the vector field $\int_{\Delta^{2}}\left[h_{t^{2}}, h_{t_{1}}\right] d t_{1} d t_{2}=\int_{0}^{1}\left[\int_{0}^{t} h_{\tau} d \tau, h_{t}\right] d t$ carries an important information, if not about the curve $\mathrm{q}(\varepsilon)$ in $\mathrm{M}$, then, at least, about the curve $\varepsilon \mapsto Q(\varepsilon)=\overrightarrow{\exp } \int_{0}^{1} \varepsilon h_{t} d t \quad$ in the group Diff(M) of diffeomorphisms. Precisely the presence of a group structure in Diff(M) leads to additional invariants, different from the tangent vector to the curve.

We consider the logical chain:

$$
\left[\int_{0}^{t} h_{\tau} d \tau, h_{t}\right]=0,0 \leqslant t \leqslant 1, \Rightarrow
$$

$\varepsilon \mapsto \overrightarrow{\exp } \int_{0}^{1} \varepsilon h_{t} d t \quad$ is a one-parameter subgroup in Diff $M \Leftrightarrow \overrightarrow{\exp } \int_{0}^{1} \varepsilon h_{t} d t=e^{e j h_{t} d t}, \varepsilon \in \mathrm{R}$, and there are no variations whatsoever, except the first one (if the first variation is equal to zero, then so are all the remaining ones). 
The first implication cannot be reversed. The condition $\left[\int_{0}^{t} h_{\tau} d \tau, h_{t}\right] \equiv 0,0 \leqslant t \leqslant 1$, is very rigid; from it there follows that all the curves $\varepsilon \mapsto \exp \int_{0}^{t} \varepsilon h_{\tau} d \tau, t \in[0,1]$, are one-parameter subgroups. It can be reversed only partially:

$$
\overrightarrow{\exp } \int_{0}^{1} \varepsilon h_{t} d t=e^{e^{1} h_{t} d t}, \varepsilon \in \mathrm{R}, \Rightarrow \int_{0}^{1}\left[\int_{0}^{t} h_{\tau} d \tau, h_{t}\right] d t=0
$$

Thus, the second variation is the first obstacle for the curve $\varepsilon \mapsto \overrightarrow{\exp } \int_{0}^{1} \varepsilon h_{t} d t$ to be a one-parameter subgroup.

4. The method of the representation and the interpretation of the second variation can be extended aiso to higher variations. Namely, the third term of the Volterra series can be represented in the form of some commutator expression of the initial nonstationary field $h_{t}$ plus a polynomial in the first two terms. Naturally, this commutator expression, calculated at the point $x_{0}$, represents the third variation. In addition, the corresponding vector field is an obstacle to the fact that the curve

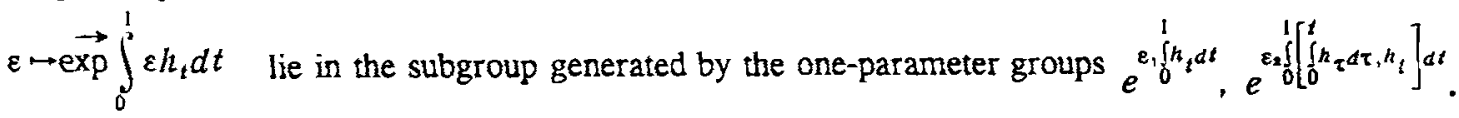

In the same way also an arbitrary n-th term of the Volterra series can be represented in the form of the sum of a vector field, namely a commutator expression of degree $n$ in $h_{t}$, and a polynomial in the first $n-1$ terms of the series. This vector field is an obstacle to the fact that the curve

$$
\varepsilon \mapsto Q(\varepsilon)=\overrightarrow{\exp } \int_{0}^{1} \varepsilon h_{1} d t
$$

lie in the group generated by the one-parameter subgroups corresponding to the previous $\mathrm{n}-1$ fields.

The simplest method to find the required commutator expressions is the following: making use of the variation formula, represent $\mathrm{Q}(\varepsilon)$ in the form of a chronological exponential with respect to $\varepsilon$ (i.e., taking the parameter $\varepsilon$ as the new "time") of some "nonstationary" (i.e., depending on $\varepsilon$ ) vector field.

Indeed,

$$
\frac{\partial}{\partial \varepsilon} Q(\varepsilon)=\overleftarrow{\omega}(\varepsilon) \bullet Q(\varepsilon)=Q(\varepsilon) \vec{\omega}(\varepsilon)
$$

where

$$
\begin{aligned}
& \overleftarrow{\omega}(\varepsilon)=\int_{0}^{1} \overrightarrow{\exp } \int_{0}^{t} \varepsilon \operatorname{ad} h_{\tau} d \tau h_{t} d t \quad(\text { see }(1.7)) \\
& \vec{\omega}(\varepsilon)=\operatorname{AdQ}^{-1}(\varepsilon) \stackrel{\leftarrow \omega}{\omega}(\varepsilon)=\int_{0}^{1} \underset{\exp }{\leftarrow} \int_{t}^{1}-\varepsilon \operatorname{ad} h_{\tau} d \tau h_{t} d t \text {. }
\end{aligned}
$$

Let

$$
\overleftarrow{\omega}(\varepsilon) \approx \sum_{n=1}^{\infty} \varepsilon^{n-1} \leftarrow^{n}, \quad \vec{\omega}(\varepsilon) \approx \sum_{n=1}^{\infty} \varepsilon^{n-1} \vec{\omega}_{n}
$$

be the expansions of $\vec{\omega}(\varepsilon)$ and $\vec{\omega}(\varepsilon)$ into series of powers of $\varepsilon$. We have

$$
\begin{gathered}
\overleftarrow{\omega}_{n}=\int \underset{\Delta^{n}}{\ldots} \ldots \int \operatorname{ad} h_{t_{n}} \ldots \operatorname{ad} h_{t_{2}} h_{t_{1}} d t_{1} \ldots d t_{n} \\
\vec{\omega}_{n}=(-1)^{n-1} \int_{\Delta^{n}}^{\ldots} \ldots \int \operatorname{ad} h_{t_{1}} \ldots \operatorname{ad} h_{t_{n-1}} h_{t_{n}} d t_{1} \ldots d t_{n} .
\end{gathered}
$$

Since 


$$
Q(\varepsilon)=\leftarrow \exp \int_{0}^{e} \omega\left(\varepsilon^{\prime}\right) d \varepsilon^{\prime}=\overrightarrow{\exp } \int_{0}^{e} \vec{\omega}\left(\varepsilon^{\prime}\right) d \varepsilon^{\prime},
$$

according to (1.3), the $\mathrm{n}$-th term of the expansion of $\mathrm{Q}(\varepsilon)$ into a series of powers of $\varepsilon$ is equal to $(1 / \mathrm{n}) \vec{\omega}_{\mathrm{n}}$ plus some polynomial of $\bar{\omega}_{1}, \ldots, \bar{\omega}_{n-1}$, and also $(1 / n) \bar{\omega}_{n}$ plus (in general, a different) polynomial of $\bar{\omega}, \ldots, \bar{\omega}_{n-1}$. Thus, both the vector field $(1 / n) \vec{\omega}_{n}$ and the field $(1 / n) \vec{\omega}_{n}$ represent the $n$-th variation of the system $(1.8)$.

For $n=1,2$ the vector fields $\vec{\omega}$ and $\bar{\omega}_{n}$ coincide; however, for $n \geq 3$ this is not so and we have two different representations of the $n$-th variation. There is nothing strange in this; in fact the admissible representation are very numerous: if to a representation of the $n$-th variation we add an arbitrary commutator polynomial of any representations of the first $n-$ 1 variations, then we obtain again a commutator expression, representing the $\mathrm{n}$-th variation. For example,

$$
\overrightarrow{\omega_{3}}=\stackrel{\leftarrow}{\omega_{3}}+\frac{1}{2}\left[\overleftarrow{\omega_{2}}, \overleftarrow{\omega_{1}}\right] \text {. }
$$

In other words, one obtains an entire Lie algebra of variations and one has only to separate that general feature which exists at various commutator representations of the same variation. A remote hint that here a meaningful theory is possible is given by the comparison of $\bar{\omega}_{n}$ with $\vec{\omega}_{n}$. One representation of the $n$-th variation is obtained from another by the inversion of the variables in the integrand and multiplication by $(-1)^{n-1}$. One of the main goals of this paper is the description of all the symmetries that transform representations of the $n$-th variation again into (in general, different) representations of the $n$-th variation.

5. We have already mentioned above that the presence of infinitesimal invariants of the curve $Q(\varepsilon)$, different from the tangent vector to this curve, are due to the fact that $\mathrm{Q}(\varepsilon)$ is a curve on a group (the group of diffeomorphisms). In this subsection we introduce the concepts, fundamental for this paper, of the variational group and the Lie algebra of variations of an arbitrary curve on the group of diffeomorphisms and we give the exact definitions of the higher variations of such a curve.

Let $R(\varepsilon), \varepsilon \in \mathbf{R}$, be a smooth curve on the group of diffeomorphisms, $R(\varepsilon) \in$ Diff $(M), \varepsilon \in \mathbf{R}, \mathbf{R}(0)=$ Id. We recall that a diffeomorphism for us is an automorphism of the algebra $C^{\infty}(M)$ (see Subsection 2). Then $\left(d^{n} / d \varepsilon^{n}\right) R(0)$ is a differential operator of order $\leq \mathrm{n}$ on $\mathrm{M}$, acting according to the rule

$$
\left.\varphi \mapsto \frac{d^{n}}{d \varepsilon^{n}}(R(\varepsilon) \varphi)\right|_{\varepsilon=0} \forall \varphi \in C^{\infty}(M)
$$

In the sequel we shall use the abbreviated notation $R^{(n)}=\left(d^{n} / d \varepsilon^{n}\right) R(0)$.

Let $n_{0}>0$ be the smallest $n$ such that $R^{(n)} \neq 0$.

It is easy to see that in this case $R^{(n o)}$ is a vector field (operator, satisfying Leibnitz' rule for the differentiation of a product). The vector field $\left(1 / \mathrm{n}_{0}\right) \mathrm{R}^{\left(\mathrm{n}_{0}\right)}$ is said to be tangent to the curve $\mathrm{R}(\varepsilon)$ at $\varepsilon=0$ and is denoted by

$$
T R(0)=\frac{1}{n_{0} !} R^{\left(n_{0}\right)}
$$

Then $\mathrm{R}(\varepsilon) \approx \mathrm{id}+\varepsilon^{\mathrm{n}_{0}} \mathrm{TR}(0)+\mathrm{O}\left(\varepsilon^{\left.\mathrm{n}_{0}\right)+1}\right)$. The number $\mathrm{n}_{0}$ is called the order of tangency of the field $\mathrm{TR}(0)$ of the curve $\mathrm{R}(\varepsilon)$,

$$
n_{0}=\operatorname{ord}(T R(0))
$$

We set further

$$
\rho(\varepsilon)=\left(\frac{d}{d \varepsilon} R(\varepsilon)\right) R(\varepsilon)^{-1} \in \operatorname{Vect}(M)
$$

and

$$
\rho^{(n)}=\frac{d^{n}}{d 8^{n}} \rho(0) \frac{1}{n !}, \quad n \geqslant 0
$$

The collection of all curves in the group Diff(M) is itself a group if the product of curves is defined pointwise: $\left(R^{\prime} \circ R^{\prime \prime}\right)(\varepsilon) \stackrel{\text { def }}{=} R^{\prime}(\varepsilon) \cdot R^{\prime \prime}(\varepsilon)$.

By $\mathscr{R}$ we denote the subgroup of the group of curves on $\operatorname{Diff}(\mathrm{M})$, generated by the curves $\varepsilon \mapsto R\left(\alpha \varepsilon^{n}\right), \alpha \in R, n=1,2, \ldots$ 
PROPOSITION 1. The following two sets coincide:

1) $\{T S(0) \mid S(\cdot) \in \mathscr{R}\}$;

2) $\operatorname{Lie}\left\{\rho^{(n)}, n \geq 0\right\}$, i.e., the Lie subalgebra in $\operatorname{Vect}(M)$, generated by the fields $\rho^{(n)}$.

Proof. First we prove that

$$
\{T S(0) \mid S(\cdot) \in \mathscr{R}\} \subset \text { Lie }\left\{\rho^{(n)}, n \geqslant 0\right\}
$$

We set

$$
\overleftarrow{\ln } S(\varepsilon)=\left(\frac{d}{d \varepsilon} S(\varepsilon)\right) \circ S(\varepsilon)^{-1} \in \operatorname{Vect}(M)
$$

Then

$$
S(\varepsilon)=\underset{\exp }{\leftarrow} \int_{0}^{e} \ln S(\tau) d \tau \quad \forall S(\varepsilon)
$$

and

$$
\overleftarrow{\ln } R(\alpha \varepsilon)=\alpha \rho(\alpha \varepsilon)
$$

The inclusion (1.11) is obtained from the following two statements, the first of which is a consequence of the Volterra expansion (1.3), while the second one is a consequence of the variation formula (1.6) and of the expansion (1.5).

i) $\frac{\varepsilon}{n} \ln S(\varepsilon)-\varepsilon^{n} T S(0)=O\left(\varepsilon^{n+1}\right)$, where $\mathrm{n}=$ ord $\mathrm{TS}(0)$ for any smooth curve $\mathrm{S}(\varepsilon)$.

ii) $\overleftarrow{\ln }\left(S_{1}(\varepsilon) \circ S_{2}^{-1}(\varepsilon)\right)=\overleftarrow{\ln } S_{1}(\varepsilon)-\exp \int_{0}^{e}-\operatorname{ad} \ln S_{1}(\tau) d \tau \overleftarrow{\ln } S_{2}(\varepsilon)$

We proceed to the proof of the inclusion

$$
\text { Lie }\left\{\rho^{(n)}, n \geqslant 0\right\} \subset\{T S(0) \mid S(\cdot) \in \mathscr{R}\} \text {. }
$$

First of all we show that the set, situated in the right-hand side of this inclusion, is a Lie subalgebra in Vect(M). The latter is a consequence of the fact that $\mathscr{R}$ is a certain group of curves. Indeed,

$$
\begin{gathered}
T\left(S_{1} \circ S_{2}\right)(0)=T S_{1}(0)+T S_{2}(0), T S^{-1}(0)=-T S(0), \\
T S(\alpha \cdot 0)=\alpha^{0 \operatorname{ord} S(0)} T S(0),
\end{gathered}
$$

where by $\operatorname{TS}(\alpha \cdot 0)$ we denote the tangent field to the curve $\varepsilon \rightarrow \mathrm{S}(\alpha \varepsilon)$; finally, if ord $\mathrm{TS}_{1}(0)=$ ord $\mathrm{TS}_{2}(0)=\mathrm{n}$, then

$$
\begin{aligned}
& T\left(S_{1} \circ S_{2} \circ S_{1}^{-1} \circ S_{2}^{-1}\right)(0)=\left[T S_{1}(0), T S_{2}(0)\right] \\
& \quad \text { ord } T\left(S_{1} \circ S_{2} \circ S_{1}^{-1} \circ S_{2}^{-1}\right)(0)=2 n .
\end{aligned}
$$

It remains to prove that $\rho^{(\mathrm{n})} \in\{\mathrm{TS}(0) \mid \mathrm{S}(\cdot) \in \mathscr{R}\}$, knowing already that $\{\mathrm{TS}(0) \mid \mathrm{S}(\cdot) \in \mathscr{R}\}$ is a Lie subalgebra in Vect(M).

We have $\rho^{(0)}=\operatorname{TR}(0)$. Further,

$$
\overleftarrow{\ln }\left(R\left(\frac{\varepsilon}{2}\right) \cdot R^{-1}(\varepsilon) \cdot R\left(\frac{\varepsilon}{2}\right)\right)=\sum_{k=1}^{\infty}\left(\left(2^{-k}-1\right) \rho_{k}+\pi_{k}^{1}\left(\rho_{0}, \ldots, \rho_{k-1}\right)\right) \varepsilon^{k},
$$

where $\pi_{k}^{1}$ is some commutator polynomial of $\rho_{0}, \ldots, \rho_{k-1}, k=1,2, \ldots$ This follows easily from the variation formula and the expansion (1.5). Consequently, the tangent field to the curve $\varepsilon \rightarrow R(\varepsilon / 2) \circ R^{-1}(\varepsilon) \circ R(\varepsilon / 2)$ is equal to $-1 / 4 \rho_{1}$ (see i)). We continue this in the same spirit, making use of induction on the tangency order. Let $S(\cdot) \in \mathscr{R}$ be a curve such that $\overleftarrow{\text { In }} S(\varepsilon)=$ $\sum_{k=n-1}^{\infty} 8^{k} \theta_{k-1}$ Then

$$
\underset{\ln }{\leftarrow}\left(S\left(2^{-\frac{1}{n}} \varepsilon\right) \cdot S^{-1}(\varepsilon) s S\left(2^{-\frac{1}{n}} \varepsilon\right)\right)=\sum_{k=n}^{\infty}\left(\left(2^{\frac{n-k-1}{n}}-1\right) \vartheta_{k}\right)+\pi_{k}^{n}\left(\vartheta_{n-1} \ldots \ldots \vartheta_{k-1}\right) \varepsilon^{k}
$$


where $\pi_{\mathrm{k}}^{\mathrm{n}}$ is a commutator polynomial of $\vartheta_{\mathrm{n}-1}, \ldots, \vartheta_{\mathrm{k}-1}$. In particular, the tangent field to the curve $\varepsilon \rightarrow S\left(2^{-\frac{1}{n}} \varepsilon\right) \circ S^{-1}(\varepsilon) \rho S$. $\left(2^{-\frac{1}{n}} \varepsilon\right)$ is equal to $\frac{2^{-\frac{1}{n}}-1}{n+1} \vartheta_{n}$ (see i)).

Definition. By the variational group of the curve $\varepsilon \rightarrow R(\varepsilon)$ in Diff(M) we mean the subgroup in Diff(M) that is generated by the diffeomorphisms $\mathbf{R}(\varepsilon), \varepsilon \in \mathbf{R}$, i.e., by the "points" of the curve $\mathbf{R}(\cdot)$.

We note that the variational group of the curve $R(\cdot)$ coincides with $\{S(\varepsilon) \mid S(\cdot) \in \mathscr{R}\}$ for any $\varepsilon \in \mathbf{R}$.

Definition. By the Lie algebra of variations of the curve $\varepsilon \rightarrow \mathrm{R}(\varepsilon)$ we mean the Lie subalgebra $\{\operatorname{TD}(0) \mid \mathrm{S}(\cdot) \in \mathscr{R}\}$ in $\operatorname{Vect}(\mathrm{M})$.

Remark. In the case when the curve $\mathrm{R}(\cdot)$ is such that its variational group is a finite-dimensional Lie group, while $\mathrm{R}(\cdot)$ itself is an analytic curve on this group, the Lie algebra of variations is simply the Lie algebra of the variational group.

Meanwhile, the Lie algebra of variations is correctly defined also in the general infinite-dimensional situation, when the standard Lie theory does not work.

We also mention that variational groups and Lie algebras of variations can be naturally defined and satisfactorily described, not only for curves in $\operatorname{Diff}(\mathbf{M})$, but also for mappings of $\mathbf{R}^{\mathbf{k}}$ into $\operatorname{Diff}(\mathbf{M}), \mathbf{k}>1$. However, in this paper these objects are not used and we postpone their description to subsequent publications.

Taking into account, not only the tangent fields to the curves from $\mathscr{R}$, but also the orders of tangency, we obtain a natural nondecreasing filtration of the Lie algebra of variations by Lie subalgebras, generated by fields that have order of tangency at most $n, n=1,2, \ldots$. The analysis of the proof of Proposition 1 shows that we have the following

PROPOSITION 2. For any $n \geq 1$ we have the equality

$$
\operatorname{Lie}\{T S(0) \mid S(\cdot) \in \mathscr{R}, \operatorname{ord} T S(0) \leqslant n\}=\operatorname{Lie}\left\{\rho^{(0)}, \ldots, \rho^{(n-1)}\right\}
$$

Now, finally, we are ready to give the precise definition of the higher variations of a curve $R(\cdot)$.

Definition. By the variation of order $n>0$ of a curve $\varepsilon \rightarrow R(\varepsilon)$ in Diff(M) we mean the image of the vector field $(1 / n) \rho^{(n-1)}$ under the canonical factorization

$$
\begin{gathered}
\{T S(0) \mid S(\cdot) \in \mathscr{R}\} \rightarrow\{T S(0) \mid S(\cdot) \in \mathscr{R}\} / \operatorname{Lie}\{T S(0) \mid S(\cdot) \in \mathscr{R} \\
\text { ord } T S(0)>n\} .
\end{gathered}
$$

Let $x \in M$ and let $n(x)$ be the smallest $n$ such that $\left(d^{n} / d \varepsilon^{n}\right) x \cdot R(0) \neq 0$. Then

$$
x \circ R(\varepsilon)=x+\frac{\varepsilon^{n}}{n(x)} \rho^{(n(x)-1)}+O\left(\varepsilon^{n+1}\right)
$$

At the same time, $\mathrm{x} \circ \rho^{(\mathrm{k})}=0$ for $\mathrm{k}<\mathrm{n}-1$ and, consequently,

$$
\begin{gathered}
0=x \circ \operatorname{Lie}\left\{\rho^{(0)}, \ldots, \rho^{n(x)-2}\right\}= \\
=x \operatorname{Lie}\{T S(0) \mid S(\cdot) \in \mathscr{R}, \text { ord } T S(0)<n(x)\} .
\end{gathered}
$$

These relations elucidate the definition of the higher variations.

With this we conclude the consideration of an arbitrary curve in the group of diffeomorphisms and we return to the perturbations of dynamical systems and to the asymptotic expansions generated by them (see (1.1), (1.8), (1.9)). The variational group, the Lie algebra of variations, and the variations of the system (1.8) (and also of the system (1.1)) are defined as the corresponding objects for the curve

$$
\varepsilon \leftrightarrow \overrightarrow{\exp } \int_{0}^{1} \varepsilon h_{t} d t \text { in } \operatorname{Diff}(M)
$$




\section{THE SHUFFLE ALGEBRA. THE ABSTRACT VARIATIONAL GROUP AND THE ABSTRACT LIE ALGEBRA OF VARIATIONS}

1. During an unsystematic investigation of variational groups and of Lie algebras of variations, one has to consider products of several Volterra series of the form (1.3). In order to work with some objects one makes use of the so-called shuffle multiplication.

Let $p_{n}:\left(t_{1}, \ldots, t_{n}\right) \rightarrow p_{n}\left(t_{1}, \ldots, t_{n}\right), n=0,1,2, \ldots$, be locally summable mappings from $R^{n}$ into the associative algebra of differential operators onto $M$. The formal power series in the variable s,

$$
p_{0}+\sum_{n=1}^{\infty} s^{n} \int \ldots \int p_{n}\left(t_{1}, \ldots, t_{n}\right) d t_{1} \ldots d t_{n}
$$

is called a chronological series (defined by the sequence $p_{n}$ ).

The product of several chronological series contains already integrals over products of simplexes:

$$
\begin{aligned}
& \left(\sum_{n=0}^{\infty} s^{n} \int \ldots \int p_{\Delta^{n}}^{1}\left(t_{1}, \ldots, t_{n}\right) d t_{1} \ldots d t_{n}\right) \circ \ldots\left(\sum_{n=0}^{\infty} s^{n} \int \underset{\Delta^{n}}{\int} \int p_{n}^{l}\left(t_{1}, \ldots, t_{n}\right) d t_{1} \ldots d t_{n}\right)= \\
& =\sum_{n=0}^{\infty} s^{n} \sum_{k_{1}+\ldots+k_{l}=n} \int_{\Delta^{k_{1}} \times . . \times \Delta^{\prime}} \ldots \int_{k_{l}} \otimes_{i=1}^{l} p_{k_{l}}^{l}\left(t_{1}, \ldots, t_{n}\right) d t_{1} \ldots d t_{n}
\end{aligned}
$$

where

$$
\bigotimes_{l=1}^{\ell} p_{k_{l}}^{l}\left(t_{1}, \ldots, t_{n}\right)=p_{k_{1}}^{l}\left(t_{1}, \ldots, t_{k_{1}}\right) \ldots \circ p_{k_{l}}^{l}\left(t_{n-k_{l-1}}, \ldots, t_{n}\right)
$$

In order to represent such a series in the form of a chronological series, it is sufficient to take triangulations of the products of several simplexes, without adding new vertices, and then to map linearly each simplex of the triangulation onto the standard simplex by performing an appropriate substitution of the integration variables.

Let $\Sigma_{n}$ be the group of all permutations of the n-element set and let $T \subset\{1, \ldots, n\}$. We introduce the notation

$$
S_{n}(T)=\left\{\sigma \in \Sigma_{n} \mid \sigma(i)<\sigma(i+1) \forall i \in\{1, \ldots, n\} \backslash T\right\} \text {. }
$$

We shall use also the simplified notation

$$
S_{n}\left(k_{1}, \ldots, k_{l}\right)=S_{n}\left(\left\{k_{1}, k_{1}+k_{2}, \ldots, n-k_{l}\right\}\right)
$$

where $\mathrm{n}=\sum_{i=1}^{l} \mathrm{k}_{\mathrm{i}}$.

Finally, for any permutation $\sigma \in \Sigma_{n}$ and vector-valued function $q\left(t_{1}, \ldots, t_{n}\right)$ we set $\sigma q\left(t_{1}, \ldots, t_{n}\right)=q\left(t_{\sigma(1)}, \ldots, t_{\sigma(n)}\right)$. It is easy to see that $(\nu \circ \sigma) q=\nu(\sigma q)$, where $\nu \circ \sigma$ is the product (composition) of permutations.

One can show that

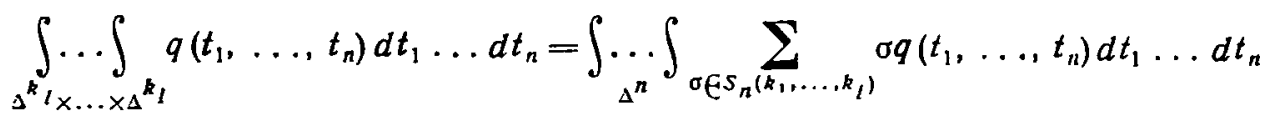

for any summable $q$.

Definition. By the shuffle product of the vector-valued functions $p_{k}{ }^{\prime}\left(t_{1}, \ldots, t_{k}\right)$ and $p_{n-k}{ }^{\prime \prime}\left(t_{1}, \ldots, t_{n-k}\right)$ we mean the vector-valued function

$$
p_{k}^{\prime} w p_{n-k}^{*}\left(t_{1}, \ldots, t_{n}\right)=\sum_{\sigma \in S_{n}(k)} \sigma\left(p_{k}^{\prime} \otimes p_{n-k}^{*}\right)\left(t_{1}, \ldots, t_{n}\right)
$$

It is easy to show that the shuffle multiplication is an associative operation and, moreover,

$$
\left.p_{k_{1}}^{l} \mathrm{w} \ldots w p_{k_{l}}^{l}=\sum_{\sigma \in S_{n}\left(k_{1} \ldots, k_{l}\right)} \underset{l=1}{\sigma(\otimes} p_{k_{l}}^{l}\right)
$$


$\forall \mathrm{k}_{1}, \ldots, \mathrm{k}_{l}$ such that $\sum_{i=1}^{l} \mathrm{k}_{\mathrm{i}}=\mathrm{n}$.

2. Diverse variants of the shuffle multiplication have been encountered, starting from the fifties, for different reasons and at several authors (also at the authors of this paper [1]). Some references can be found in the recent paper [5]. Apparently, the notation "แ" is due to M. Fliess [6]. The most important properties of the shuffle multiplication are determined, naturally, by the linear combinations of permutations, occurring in its definition, and not by the vector-valued functions $p_{n}$. At the basis of everything is the "abstract shuffle multiplication," the definition of which is given now.

Let $\subseteq$ be the group algebra of the symmetric group $\Sigma_{\mathrm{n}}$ over $\mathbf{R}, \mathrm{n}=1,2, \ldots, \subseteq=\mathbf{R}$ and $\subseteq=\oplus_{n=0}^{\infty} \Im_{n}$. By the abstract shuffle product of the permutations $\nu \in \Sigma_{\mathrm{k}}$ and $\nu^{\prime} \in \Sigma_{\mathrm{k}^{\prime}}$ we mean the following element from $\Im_{k+k^{\prime}}$

$$
v \amalg v^{\prime}=: \sum_{\sigma \in S_{k+k^{\prime}(k)}} \sigma_{0}\left(\nu \otimes v^{\prime}\right), \text { where }\left(v \otimes v^{\prime}\right)(i)=\left\{\begin{array}{l}
v(i), i \leqslant k, \\
v^{\prime}(i-k)+k, i>k .
\end{array}\right.
$$

Since the permutations form an additive basis of the space $\mathfrak{S}$, the shuffle multiplication is uniquely extended "by linearity" to any pair of elements from $\subseteq$ and defines in $\mathcal{S}=\bigoplus_{n=0}^{\infty} \mathcal{S}_{n}$ a structure of graded associative algebra. Moreover, the shuffle multiplication can be extended uniquely "by continuity" to an associative multiplication in the completion $\bar{\Phi}$ of the graded space 5. As usually, by the completion of a graded space we mean the space of formal series of the form $\sum_{n=0}^{+\infty} a_{n}, a_{n} \in \mathbb{S}_{n}$, while multiplication is performed according to Cauchy:

$$
\left(\sum_{n=0}^{\infty} a_{n}\right) \Psi\left(\sum_{n=0}^{\infty} b_{n}\right)=\sum_{n=0}^{\infty}\left(\sum_{k=0}^{n} a_{k} \omega b_{n-k}\right) .
$$

The algebra $\mathfrak{S}$, just as other algebra of formal series, is considered with the standard topology of termwise convergence. The algebra $\overline{\mathfrak{S}}$ is indeed the completion of $\mathcal{S}$ in this topology, while the multiplication "according to Cauchy" is the extension by continuity of the shuffle multiplication in $\Xi$.

Everywhere in the sequel, unless otherwise mentioned, by an associative algebra we mean an associative algebra with identity over $\mathbf{R}$. For each associative algebra $\mathfrak{A}$, by $[\mathfrak{A}]$ we denote its associated Lie algebra with Lie multiplication $[a, b]$ $=a b-\mathrm{b} a, \mathrm{~b} \in \mathfrak{A}$.

3. We recall the nonstationary vector field $h_{t}$ in which we were interested in the first section. The mapping

$$
h_{*}: v \mapsto s^{n} \int_{\Delta^{n}}^{0} \ldots \int h_{t_{v(n)}} \ldots \circ h_{t_{v(1)}} d t_{1} \ldots d t_{n}, \quad v \in \Sigma_{n}, \quad n>0
$$

can be extended in a unique manner to a continuous homomorphism $\overline{\mathrm{h}}$. of the algebra $\bar{\Phi}$ with shuffle multiplication into the algebra of chronological series.

For $\mathrm{n}=1,2, \ldots$ we denote by $\mathbf{1}_{\mathrm{n}}$ the identity element in the group $\Sigma_{\mathrm{n}}, 1_{0}=1 \in \mathbf{R}$. We have

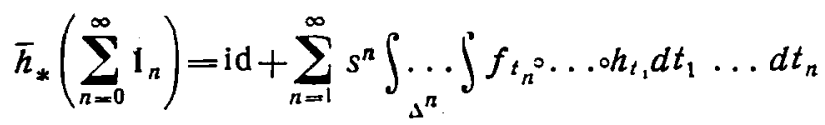

the asymptotic expansion of the right chronological exponential. We introduce the notation $\overrightarrow{\exp }(\varepsilon)=\sum_{n=0}^{\infty} \varepsilon^{n} 1_{n}, \varepsilon \in \mathbf{R}$. The elements $\operatorname{ex} \mathrm{p}(\varepsilon)$ are invertible in $\bar{\varsigma}$ as any formal series with invertible constant terms.

Definition. The group $\mathscr{\gamma}$, generated by the elements $\exp (\varepsilon), \varepsilon \in \mathbf{R}$, with the multiplication operation "w", is called an abstract variational group. 
According to definition, $\mathscr{P} \subset \bar{\complement}$. The curves with values in the group $\mathscr{P}$ (just as in any other group) form themselves a group with pointwise multiplication. We denote by $\mathscr{E}$ the subgroup in the group of the curves on $\mathscr{E}$, generated by the curves

$$
\left.\varepsilon \mapsto \overrightarrow{\exp }\left(\alpha^{k} \varepsilon\right), \quad \alpha \in \mathbf{R}, k=1,2, \ldots \text { (see Subsection } 1.5\right)
$$

An arbitrary curve $a(\cdot) \in \mathscr{E}$ has the form $a(\varepsilon)=1+\sum_{n=1}^{\infty} \varepsilon^{\mathrm{n}} a_{\mathrm{n}}, \varepsilon \in \mathbf{R}$, where $a_{n} \in \mathcal{S}, \quad n=1,2, \ldots$

Let $\mathrm{n}_{0}$ be the smallest $\mathrm{n}$ such that $a \neq 0$. We denote $a_{\mathrm{n}_{0}}=\mathrm{T} a(0)$; this is the abstract analogue of the tangent field to a curve on the group of diffeomorphisms. In accordance with the notations of Sec. 1, we set

$$
\stackrel{\omega}{\omega}(\varepsilon)=\sum_{n=1}^{\infty} \varepsilon^{n-1} \hat{\omega}_{n}=\frac{\partial}{\partial \varepsilon}(\overrightarrow{\exp }(\varepsilon)) \omega(\overrightarrow{\exp }(\varepsilon))^{-1}
$$

Just as Proposition 1, one proves the following

PROPOSITION. The following two subsets in 5 coincide:

1) $\{\operatorname{Ta}(0) \mid a(\cdot) \in \mathbb{E}\}$

2) Lie $\left\{\omega_{n} \mid n \geqslant 1\right\}$ i.e., the Lie subalgebra in [S] , generated by the elements $\overleftarrow{\omega}_{n} \in S_{n}, n=1,2, \ldots$

Definition. The Lie subalgebra

$$
V=\{\operatorname{Ta}(0) \mid a(\cdot) \in \mathscr{E}\} \text { in }[S]
$$

is called the abstract Lie algebra of variations.

The abstract variational group and the abstract Lie algebra of variations lie in the closed associative subalgebra of $\overline{\mathfrak{S}}$, generated by the elements $1_{n}, n \geq 0$.

Definition. The associative subalgebra in $€$, generated by the elements $1_{n}, n \geq 0$, is called the shuffle algebra and

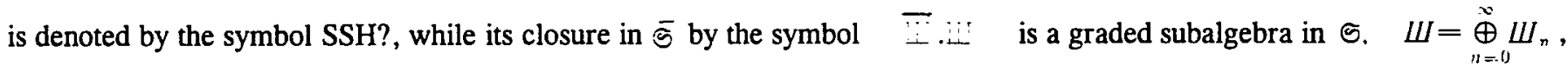
where $W_{n}=W_{\cap} \cap \Theta_{n}$.

4. When working with the shuffle algebra, the following concepts, related to the combinatorics of permutations, are useful.

Definition. By the monotonicity type of an arbitrary permutation $\sigma \in \Sigma_{\mathrm{n}}$ we mean the word $S(\sigma)$ of length $\mathrm{n}-1$, $S(\sigma)=s_{1} \ldots s_{n-1}$, in the alphabet of two letters $\alpha, \beta$, defined by the following rule:

$$
s:= \begin{cases}\alpha, & \text { if } \quad w(i)<\sigma(i+1) \\ \beta, & \text { if } \quad \sigma(i)>\sigma(i+1)\end{cases}
$$

For example, $S\left(1_{n}\right)==\underbrace{\alpha \ldots}_{n-1} \ldots=\nu^{n-i} \quad$, while the monotonicity type of the inversion $\mathrm{i} \rightarrow \mathrm{n}-\mathrm{i}+1, \mathrm{i}=1, \ldots, \mathrm{n}$, is the word $\underbrace{\beta \ldots \beta}_{n-1}=\beta^{n-1} ;$ for $\mathrm{n}=1$ we obtain in both cases the empty word.

The sum of all permutations having the same monotonicity type $a$ will be denoted by iaj. If $a$ is a word of length $n$ -1 , then $\mathrm{i} a \mathrm{j} \in \mathfrak{S}_{n}$. The notation $\mathrm{i} a \mathrm{j}$ may seem to be strange at the first glance, but it will be elucidated a little later.

PROPOSITION 3. Let $a$ be a word of length $\mathrm{n}-1$ in the alphabet of the letters $\alpha, \beta$, and let $\mathrm{T}_{a} \subset\{1, \ldots, \mathrm{n}-1\}$ be the subset consisting of the indices of the "places" in the word $a$ where the letter $\beta$ occurs, \# $\mathrm{T}_{a}=\mathrm{m}$. Then

$$
i a j=\sum_{k=0}^{m}(-1)^{m-k} \sum_{\left\{i_{1}, \ldots, l_{k}\right\} \subset \tau_{a}} 1_{i_{1}} w 1_{i_{2}-l_{1}} w \ldots w \mathbf{1}_{i_{k}-i_{k-1}} w \mathbf{1}_{n-l_{k}}
$$

Proof. According to definition,

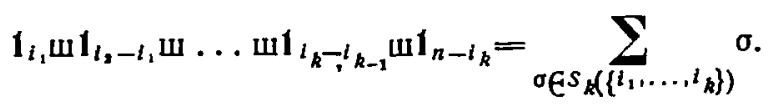

Consequently, the element (2.1) is the sum of all permutations, whose monotonicity type contains the letter $\alpha$ at all the places, except at $i_{1}, \ldots, i_{k}$, while at the places $i_{1}, \ldots, i_{k}$ one can have either the letter $\alpha$ or $\beta$. Thus, in the sum (2.1), each 
permutation, having monotonicity type $a$, occurs with coefficient 1 , while a permutation, containing the letter $\beta$ exactly at the places $\mathrm{j}_{1}, \ldots, \mathrm{j}_{l}$, where $\left\{\mathrm{j}_{1}, \ldots, \mathrm{j}_{l}\right\}$ is contained strictly in $\mathrm{T}_{a}$, occurs with the coefficient $\sum_{k=1}^{m}(-1)^{\mathrm{m}-\mathrm{k}} \mathrm{C}_{\mathrm{m}-l} l^{\mathrm{k}-l}=0=0$. Indeed, $\left\{\mathrm{j}_{1}, \ldots, \mathrm{j}_{l}\right\}$ is contained exactly in $\mathrm{C}_{\mathrm{m}-l} l^{\mathrm{k}-l}$ subsets of $\mathrm{T}_{a}$ of cardinality $\mathrm{k}$.

COROLLARY. SSH? is a free associative graded algebra, having exactly one generator of each degree $n>0$. The elements of the form iaj, where $a$ is an arbitrary word in the alphabet $\alpha, \beta$, form an additive basis of the algebra $\amalg$.

Proof. From Proposition 1 there follows that $\mathrm{i}$ for any word $a$ of length $\mathrm{n}-1$. Obviously, the elements iaj $\in \Psi_{\mathrm{n}}$ for distinct words $a$ are linearly independent. Since one has in all $2^{n-1}$ words of length $n-1$, it follows that dim $\amalg_{n} \geq$ $2^{n-1}$. On the other hand, the algebra $W$ is generated by the elements $1_{n}, n \geq 0$; consequently, it has at most one generator of each positive degree. It remains to note that the dimension of the components of degree $n$ for a free associative algebra, having one generator of each positive dimension, is equal exactly to $2^{\text {n-1 }}$.

THEOREM 1. The abstract Lie algebra of variations $V$ is a free Lie algebra with generators $\bar{\omega}_{n} \in S S H ? n, n=1$, $2, \ldots$

Proof. We have

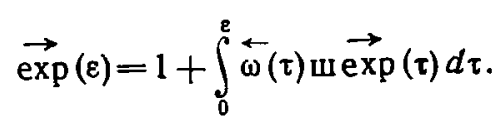

Consequently,

$$
1_{n}=\frac{1}{n} \sum_{k=1}^{n} \overleftarrow{\omega}_{k} \omega 1_{n-k}, \quad n=1,2, \ldots
$$

From the formulas (2.3) we derive by induction on $n$ that the elements $\bar{\omega}_{n} \in{ }_{i}{ }_{n}$, generate the algebra $\ldots$. Since $\stackrel{\cdots}{\cdots}$ is a free algebra, having one generator in each of the homogeneous components $i_{n}$, it follows that $\overleftarrow{\omega}_{n} \geq 1$, are free generators of the associative algebra $\ldots$. From standard result of the theory of Lie algebras there follows that $\bar{\omega}_{n}, n \geq 1$, are free generators of the Lie subalgebra in $\cdots$, generated by these elements.

COROLLARY. The imbedding $\mathrm{V} \subset \ldots$ induces an isomorphism of the universal enveloping algebra $U V$ of the Lie algebra $\mathrm{V}$ onto the algebra - , i.e., $\approx \cdots$.

Indeed, the universal enveloping algebra of a free Lie algebra is a free associative algebra with the same collection of generators.

Remark. Certainly, the sequence $\overleftarrow{\omega}_{\mathrm{n}}, \mathrm{n} \geq 1$, is not the unique system of homogeneous generators of the Lie algebra V. As another system of homogeneous generators of the same Lie algebra we can take, for example, $\vec{\omega}_{n}$, $n \geq 1$, where $\sum_{n=1}^{\infty} \varepsilon^{n-1} \vec{\omega}_{n}=\vec{\omega}(\varepsilon)=(\exp (\varepsilon))^{-1} w \frac{\partial}{\partial s}(\overrightarrow{\exp }(\varepsilon))$. In the next section we give an explicit description of all possible systems of generators.

5. Thus, $i \quad$ is the subalgebra in $\sigma$, consisting of all those linear combinations of permutations such that the permutations with the same monotonicity type have the same coefficients.

PROPOSITION 4. For any words $a, \mathrm{~b}$ in the alphabet $\alpha, \beta$ we have the equality

$$
(i a j) ш(i b j)=i a \alpha m j+i a \beta b j .
$$

Proof. Let $i a j \in \Psi_{n}$, ibj $\in I_{m}$. Then

$$
(i a j) w(i b j)=\sum_{\sigma \in s_{n+m^{(n)}}} \sigma \alpha(i a j \otimes i b j) .
$$

Since each permutation $\sigma \in S_{n+m}(n)$ increases monotonically on the segment $1, \ldots, n$ and, separately on the segment $n+1$, $\ldots, \mathrm{n}+\mathrm{m}$, it follows that $\forall \mu \in \Sigma_{\mathrm{n}}, \nu \in \Sigma_{\mathrm{m}}$ the monotonicity type $\mathrm{S}\left(\sigma_{\circ}(\mu \otimes \nu)\right)$ of the permutation $\sigma \circ(\mu \otimes \nu)$ is equal either to $S(\mu) \alpha S(\nu)$ or to $S(\mu) \beta S(v)$. Consequently,

$$
(i a j) w(i b j)=k_{1}(i a a b j)+k_{2}(i a \beta b j),
$$


where $k_{1}, k_{2}$ are nonnegative integers. Further, if $\sigma \in S_{n+m}(n)$ and $\sigma o\left(\mu_{1} \otimes v_{1}\right)=\mu_{2} \otimes v_{2}$, then $\sigma=\mu_{1}{ }^{-1} \circ \mu_{2} \otimes \nu_{1}-1 \circ \nu_{2}$ $=1_{n+m}$ since $S_{n+m}(n) \cap\left(\Sigma_{n} \otimes \Sigma_{m}\right)=1_{n+m}$. Consequently, the coefficients $k_{1}, k_{2}$ are equal either to zero or to unity. In order to conclude the proof it remains to note that

$$
\begin{gathered}
S\left(1_{n+m} \circ(\mu \otimes v)\right)=S(\mu \otimes v)=S(\mu) \alpha S(v), \\
S((1, \ldots, n-1, n+1, n, n+2, \ldots, n+m) \circ(\mu \otimes v))=S(\mu) \beta S(v) .
\end{gathered}
$$

By the symbol $\mathfrak{A}$ we denote the associative algebra with generators $\alpha, \beta, \mathrm{i}, \mathrm{j}$, not free, but defined by the relations

$$
j i=\alpha+\beta, i^{2}=j^{2}=\alpha i=\beta i=j \alpha=j \beta=0 .
$$

We define in the algebra $\mathfrak{A}$ the grading by semiintegers $\mathfrak{X}=\bigoplus_{k=0}^{\infty} \mathfrak{A}_{k}$, assigning to the variables the following degrees: deg $\alpha$ $=\operatorname{deg} \beta=1, \operatorname{deg} \mathbf{i}=\operatorname{deg} \mathbf{j}=1 / 2$. From Proposition 4 there follows that $U \approx \bigoplus_{n=0}^{\infty} \mathfrak{A}_{n}$, i.e., the graded algebra SSH? can be identified in a natural manner with the subalgebra in $\mathfrak{A}$, consisting of the linear combinations of homogeneous elements of integer degree. We shall make use of this identification without any special reference up to the end of this section, i.e., we shall assume that $\amalg \subset \mathfrak{A}$ (while $\bar{W} \subset \overline{\mathfrak{A}}$ ), and we shall omit the symbol sh? at the multiplication of the elements a shuffle algebra, represented in the form of polynomial of the (noncommutative) variables $\alpha, \beta, \mathrm{i}, \mathrm{j}$.

We recall that

$$
\begin{gathered}
\overrightarrow{\exp }(\varepsilon)=\sum_{n=0}^{\infty} \varepsilon^{n} 1_{n}=1+\varepsilon \sum_{n=0}^{\infty} \varepsilon^{n} i \alpha^{n} j=1+\varepsilon i(1-\varepsilon \alpha)^{-1} j \\
\overleftarrow{\omega}(\varepsilon)=\sum_{n=1}^{\infty} \varepsilon^{n-1} \overleftarrow{\omega}_{n}=\left(\frac{\partial}{\partial \varepsilon} \overrightarrow{\exp }(\varepsilon)\right) w(\overrightarrow{\exp }(\varepsilon))^{-1} \\
\vec{\omega}(\varepsilon)=\sum_{n=1}^{\infty} \varepsilon^{n-1} \vec{\omega}_{n}=(\overrightarrow{\exp }(\varepsilon))^{-1} w \frac{\partial}{\partial \varepsilon} \overrightarrow{\exp }(8) .
\end{gathered}
$$

PROPOSITION 5. We have the identities

1) $(\exp (\varepsilon))^{-J}=1-\varepsilon i(1+\varepsilon \beta)^{-1} j=1-\varepsilon \sum_{n=0}^{\infty}(-\varepsilon)^{n} i \beta^{n} j$

2) $\overleftarrow{\omega}(\varepsilon)=i(1-\varepsilon \alpha)^{-1}(1+\varepsilon \beta)^{-1} j$

3) $\vec{\omega}(\varepsilon)=i(1+\varepsilon \beta)^{-1}(1-\varepsilon \alpha)^{-1} j$.

Proof.

1) $\left(1+\varepsilon i(1-\varepsilon \alpha)^{-1} j\right)\left(1-\varepsilon i(1+\varepsilon \beta)^{-1} j\right)=1+\varepsilon i\left((1-\varepsilon \alpha)^{-1}-(1+\varepsilon \beta)^{-1}\right) j-\varepsilon i(1-\varepsilon \alpha)^{-1}(\varepsilon \alpha+\varepsilon \beta)(1+\varepsilon \beta)^{-1} j=1$

2) $\leftarrow(\varepsilon)=\left(\frac{\partial}{\partial \varepsilon}\left(\varepsilon i(1-\varepsilon \alpha)^{-1} j\right)\right)\left(1-\varepsilon i(1+\varepsilon \beta)^{-1} j\right)=i(1-\varepsilon \alpha)^{-2} j \times\left(1-\varepsilon i(1+\varepsilon \beta)^{-1} j\right)=i(1-\varepsilon \alpha)^{-2}(1-(\varepsilon \alpha+\varepsilon \beta)$ $\left.(1+\varepsilon \beta)^{-1}\right) j=i(1-\varepsilon \alpha)^{-1}(1+\varepsilon \beta)^{-1} j$.

Identity 3 ) is proved in a similar manner.

COROLLARY.

$$
\overleftarrow{\omega}_{n+1}=\sum_{k=0}^{n}(-1)^{k} i \alpha^{n-k} \beta^{k} j ; \quad \vec{\omega}_{n+1}=\sum_{k=0}^{n}(-1)^{k} i \beta^{k} \alpha^{n-k} j, \quad n \geqslant 0 .
$$

6. We recall that by a differentiation in an arbitrary algebra we mean any linear operator $D$ in this algebra, satisfying the "Leibnitz differentiation rule": $D(x y)=(D x) y+x(D y)$. We define two homogeneous differentiations $u$, $v$ of degree 1 of the graded associative algebra $\mathfrak{A}$, by giving their values on the generators with the aid of the formulas

$$
\begin{aligned}
& u(\alpha)=\alpha^{2}, \quad u(\beta)=-\beta^{2}, \\
& u(i)=i \alpha, \quad u(j)=-\beta j \\
& v(\alpha)=-\alpha^{2}, \quad v(\beta)=\beta^{2}, \\
& v(i)=i \beta, \quad v(j)=-\alpha j .
\end{aligned}
$$


Straightforward computation shows that the formulas (2.4) are consistent with the identities defining the algebra $\mathfrak{A}$. For example: $\mathrm{u}(\mathrm{ji})=\mathrm{u}(\mathrm{j}) \mathrm{i}+\mathrm{ju}(\mathrm{i})=-\beta \mathrm{ji}+\mathrm{ji} \alpha=-\beta(\alpha+\beta)+(\alpha+\beta) \alpha=\alpha^{2}-\beta^{2}=\mathrm{u}(\alpha+\beta)$, etc. Therefore, the formulas (2.4) define correctly differentiations of $\mathfrak{A}$. In addition, since the operators $u, v$ are homogeneous and have integer degree, they map the subalgebra $\amalg \subset \mathfrak{A}$ into itself.

PROPOSITION 6. We have the identities

$$
\stackrel{\leftrightarrow}{\omega}(\varepsilon)=e^{e u}(i j), \quad \vec{\omega}(\varepsilon)=e^{-\varepsilon y}(i j)
$$

Proof. $\omega_{1}=\mathrm{ij}$,

$$
\begin{gathered}
u \overleftarrow{\omega}_{n+1}=u \sum_{k=0}^{n}(-1)^{k} i \alpha^{n-k} \beta^{k} j=\sum_{k=0}^{n}(-1)^{k}\left(u\left(i \alpha^{n-k}\right) \beta^{k} j+i \alpha^{n-k} u\left(\beta^{k} j\right)\right)= \\
=\sum_{k=0}^{n}(-1)^{k}(n+1-k) i \alpha^{n+1-k} \beta^{k} j-\sum_{k=0}^{n}(-1)^{k}(k+1) i \alpha^{n-k} \beta^{k+1} j= \\
=(n+1) \sum_{k=0}^{n+1}(-1)^{k} i \alpha^{n+1-k} \beta^{k} j=(n+1) \overleftarrow{\omega}_{n+2}
\end{gathered}
$$

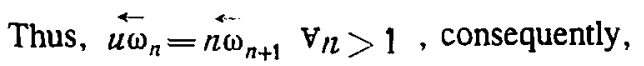

$$
\overleftarrow{\omega}_{n+1}=\frac{u^{n}}{n !} \overleftarrow{\omega_{1}}, \quad e^{\varepsilon u \overleftarrow{\omega}_{1}}=\overleftarrow{\omega}(\varepsilon) .
$$

A similar computation proves also the second identity.

The following proposition shows that the differentiations $\mathrm{u}, \mathrm{v}$ are closely related with the inner differentiations adx: $a \rightarrow \mathrm{x} a-a \mathrm{x}$ of the algebra $\mathfrak{A}$ for $\mathrm{x} \in \mathrm{V} \subset \mathfrak{A}$.

PROPOSITION 7. We have the identities

$$
\operatorname{ad} \overleftarrow{\omega}(\varepsilon)=e^{\operatorname{ead} u} v+u, \quad \operatorname{ad} \vec{\omega}(\varepsilon)=e^{-\mathrm{ead} v} u+v .
$$

Proof. For $\varepsilon=0$ both identities reduce to the equality ad(ij) $=u+v$. This equality is verified by straightforward computation: since both the right- and the left-hand sides of the equality are differentiations of the algebra $i$ i it is sufficient to verify that they coincide on the generators. The rest follows from Proposition 4 and from the universal identity $[D, a d x]=$ $\operatorname{ad}(D x)$, valid for any differentiation $D$ and any element $x$ of an arbitrary algebra.

COROLLARY. The Lie algebra generated by $u$ and $v$ is free and the mapping

$$
\text { ad : } V \rightarrow \operatorname{Der} \mathfrak{A} \text {, where ad : } x \mapsto a d x \forall x \in V \subset \mathfrak{A} \text {, }
$$

is an isomorphism of the Lie algebra $V$ onto the ideal of codimension 1 in $\operatorname{Lie}(u, v)$ of the form $R(u+v)+[\operatorname{Lie}(u, v)$, $\operatorname{Lie}(u, v)]$.

Proof. From standard results on free Lie algebras (see [4]) there follows that the subalgebra in Lie( $u, v)$, generated by the elements $u+v,(a d u)^{n} v, n \geq 1$, contains all the commutator monomials of $u, v$ of degree at least two. The rest follows from Proposition 7 and Theorem 1.

Starting from this place we digress from the operator origin of the elements $\mathrm{u}, \mathrm{v}$. Henceforth, the letters $\mathrm{u}, \mathrm{v}$ will denote the generators (alphabet) of a free associative algebra (algebra of words). We have

$$
V \approx(\mathbf{R}(u+v)+[\operatorname{Lie}(u, v), \operatorname{Lie}(u, v)]) \subset \operatorname{Lie}(u, v) \subset \operatorname{Ass}(u, v)
$$

Consequently,

$$
U=U V \approx \operatorname{Ass}\left((\operatorname{ad} u)^{n}(u+v) ; n \geqslant 0\right) \subset \operatorname{Ass}(u, v),
$$

since the elements $(\operatorname{adu})^{n}(u+v)$ are (free) generators of the Lie algebra $\mathbf{R}(u+v)+[\operatorname{Lie}(u, v), \operatorname{Lie}(u, v)]$; UV denotes the universal enveloping algebra of the Lie algebra $V$. We denote $u+v=w$. We have a simple algebraic characterization of the elements of the subalgebra Ass $\left((a d u)^{n} w, n \geq 0\right)$ in the algebra

$$
\operatorname{Ass}(u, w)=\operatorname{Ass}(u, v) \text {. }
$$


PROPOSITION 8. We consider the representation $\theta$ of the algebra Ass $(u, w)$ by linear operators in Ass( $u, w)$, defining it on the generators by the formulas

$$
\theta(u) x=[u, x], \theta(w) x=w x \quad \forall x \in \operatorname{Ass}(u, w) .
$$

Then the mapping $x \rightarrow \theta(x) 1$ is the projection of $\operatorname{Ass}(u, w)$ onto the subalgebra generated by $(a d u)^{n_{w}}, n \geq 0$.

Proof. First of all, since $\theta(u)=$ adu is a differentiation of the algebra Ass $(u, w)$, it follows that the mapping $x \rightarrow$ $\theta(x) 1$ maps Ass $(u, w)$ into Ass $\left((\operatorname{adu})^{\mathrm{n}} \mathrm{w}, \mathrm{n} \geq 0\right)$. It remains to show that this mapping is the identity on $\operatorname{Ass}\left((\operatorname{adu})^{\mathrm{n}} \mathrm{w}, \mathrm{n} \geq 1\right)$. This follows from the following statement.

LEMMA. Let $\mathrm{L}_{\mathbf{x}}$ be the operator of left multiplication by $\mathrm{x} \in \operatorname{Ass}(\mathrm{u}, \mathrm{w})$. In this case, if $\mathrm{x}$ is such that $\theta(\mathrm{x})=\mathrm{L}_{\mathrm{x}}$, then $\theta([u, x])=L_{[u, x]}$.

Proof. $\theta([u, x]) y=\theta(u) \theta(x) y-\theta(x) \theta(u) y=[u, x y]-x[u, y]=[u, x] y=L_{[u, x 1} y$.

THEOREM 2. Let $I: \bar{W} \rightarrow \overline{\operatorname{Ass}(u, v)}$ be a continuous homomorphism (and monomorphism), uniquely defined by its values on the generators $I\left(\omega_{h}\right)=(1 / n !)(a d u)^{n_{v}}+u$. Then

1) $I(\overrightarrow{\exp }(\varepsilon))=e^{\varepsilon u} e^{\varepsilon v}$.

2) $I \mid \boldsymbol{Y}^{\mathcal{D}}$ is an isomorphism of the abstract variational group $\mathscr{\gamma}$ onto the subgroup in $\overline{\operatorname{Ass}(u, v)}$, consisting of all elements of the form $e^{t_{1} u} e^{s_{1} D} \ldots e^{t_{k}} e^{s^{v}}, k>0, \sum_{i=1}^{k} t_{i}=\sum_{i=1}^{k} s_{l}$.

3) $I(V)=\mathrm{R}(u+v)+[\operatorname{Lie}(u, v)$, Lie $(u, v)]$.

Proof.

1) $\sum_{n=1}^{\infty} \varepsilon^{n-1} \stackrel{\leftarrow}{\omega_{n}}=\left(\frac{\partial}{\partial \varepsilon} \overrightarrow{\exp }(\varepsilon)\right) \omega(\exp (\varepsilon))^{-1}$. Consequently,

$$
\overrightarrow{\exp }(\varepsilon)=\exp \int_{0}^{\varepsilon} \omega\left(\varepsilon^{\prime}\right) d \varepsilon^{\prime}, \quad I(\exp (\varepsilon))=\underset{\exp }{\leftarrow} \int_{0}^{e} I\left(\omega\left(\varepsilon^{\prime}\right)\right) d \varepsilon^{\prime}=\exp \int_{0}^{e}\left(e^{e^{\prime} a d u} v+u\right) d \varepsilon^{\prime}=e^{e u} e^{e v} .
$$

We have made use of the variation formula.

2) Statement 2) follows directly from 1) and the definition of the abstract variational group.

3) Statement 3) has been already defined above and can be derived independently without difficulty from 1) and the definition of $\mathrm{V}$.

COROLLARY. The exponential mapping $\mathrm{x} \rightarrow \mathrm{e}^{\mathrm{x}}=\sum_{n=0}^{\infty}(1 / \mathrm{n} !) \mathrm{x}^{\mathrm{n}}$ maps $\overline{\mathrm{V}}$ onto $\overline{\mathscr{F}}$ in a one-to-one manner.

Proof. It is known that the exponential mapping maps $\overline{\operatorname{Lie}(u, v)}$ in a one-to-one manner onto the closed subgroup $\bar{\Gamma}$ in $\operatorname{Ass}(u, v)$, generated by the one-parameter subgroups $t \rightarrow e^{t \mathrm{u}}$ and $t \rightarrow \mathrm{e}^{\mathrm{tv}}$ (see [4]). From Theorem 2 there follows that $I(\bar{Y})$ is a normal subgroup in $\bar{\Gamma}$, consisting of all elements whose homogeneous components of degree 1 have the form $\alpha(\mathrm{u}$ $+\mathrm{v}), \alpha \in \mathbf{R}$.

\section{SHUFFLES AND SYMMETRIES}

1. In the previous section we have elucidated the structure of the shuffle algebra $i$, as well as the variational group $\overline{I I}$ and the Lie algebra of variations $\mathrm{V}$, contained in $\mathscr{Y}^{\circ}$. Now we recall that the graded algebra $U \subset \mathcal{S}=\stackrel{\infty}{\oplus \mathfrak{S}_{n}}$, where $\mathcal{G}_{n}$ is the group algebra of the symmetric group $\Sigma_{n}$ (the group of permutations of an n-element set). The elements of $\Phi_{n}$ are linear combinations of permutations, while multiplication in the group algebra is defined by the group multiplication, i.e., by the composition of permutations: if $\sigma, \nu \in \Sigma_{n}$, then also $\sigma \circ \nu \in \Sigma_{n}$. The classical multiplication " $\circ$ " will be said to be symmetric in order to avoid confusion with the shuffle multiplication "w". In addition, we extend the symmetric multiplication to all of $\Theta$, by setting $\mu \circ \nu=0$ for $\forall \mu \in \Sigma_{m}, \nu \in \Sigma_{n}, m \neq n$. It turns out that there exists a deep connection between the two associative multiplications: the shuffle product "山" and the symmetric one " $\circ$ ".

THE FUNDAMENTAL LEMMA. For any $\mathrm{x} \in \mathscr{P}$ and any $\mu, \nu \in \mathcal{F}$ we have the equality

$$
x \circ(\mu \mathrm{ur} v)=(x \circ \mu) \mathrm{w}(x \circ v) \text {. }
$$


Proof. Let $x=\sum_{k=0}^{\infty} x_{k} \in \mathcal{Y}^{\rho}$, where $x_{k} \in W_{k} \subset \mathcal{S}_{k} \forall k \geqslant 0, \mu \in \Sigma_{m}, v \in \Sigma_{n}$. One has to prove that

$$
x_{n+m} \circ(\mu \omega v)=\left(x_{m}^{\circ} \mu\right) \omega\left(x_{n}^{\circ} \circ\right)
$$

First of all, it is sufficient to prove this equality for the case $\mu=1_{m}, \nu=1_{n}$ since

$$
x_{n+m} \circ(\mu \mathrm{w} v)=x_{n+m} \mathrm{o}\left(\mathbf{1}_{m} \mathrm{w1} \mathbf{1}_{n}\right) c(\mu \otimes v)
$$

and

$$
\left(x_{m} \circ \psi(v) \mathrm{w}\left(x_{n} \circ v\right)=\left(1_{m} \mathrm{w} 1_{n}\right) \circ\left(x_{m} \circ \mu \otimes x_{n}^{\circ} \vee v\right)=\left(1_{m} \mathrm{w1} 1_{n}\right) \circ\left(x_{m} \otimes x_{n}\right) \circ(\mu \otimes v)=\left(x_{m} \mathrm{w} x_{n}\right) \circ(\mu \otimes v) .\right.
$$

Thus, the fundamental lemma is equivalent to the equalities

$$
x_{n+m}\left(1_{m} \mathrm{w} 1_{n}\right)=x_{m} \amalg x_{n} \forall_{m}, n \geqslant 0, \sum_{k=0}^{\infty} x_{k} \in \mathcal{P} \text {. }
$$

Let $J_{n} \in \Sigma_{n}$ be the inversion: $J_{n}(k)=n-k+1, k=1, \ldots, n, J_{n}=i \beta^{n-1} j$. We mention that

$$
\overrightarrow{\exp }(\varepsilon)=\sum_{n=0}^{\infty} \varepsilon^{n} 1_{n}(\exp (\varepsilon))^{-1}=\sum_{n=0}^{\infty}(-\varepsilon)^{n} J_{n},
$$

if we assume that $\mathrm{J}_{0}=1$.

An arbitrary element $\mu=\sum_{n=0}^{\infty} x_{n} \in \mathscr{P}$ has the form

$$
x=\left(\sum_{n=0}^{\infty} t_{0}^{l} a_{i}^{0}\right) \Psi\left(\sum_{i=1}^{\infty} t_{1}^{l} a_{i}^{1}\right) \Psi \ldots w\left(\sum_{i=0}^{\infty} t_{k}^{i} a_{i}^{k}\right),
$$

where for any $\mathrm{j}=0,1, \ldots, \mathrm{k}$ the sequence $a_{\mathrm{i}}^{\mathrm{j}}, \mathrm{i} \geq 0$, either coincides with $\mathbf{1}_{\mathrm{i}}, \mathrm{i} \geq 0$, or with $\mathrm{J}_{\mathrm{i}}, \mathrm{i} \geq 0 ; \mathrm{t}_{\mathrm{j}}, \mathrm{j}=0,1, \ldots, \mathrm{k}$ are arbitrary real numbers, $\mathrm{k}$ is an arbitrary nonnegative integer.

Formula (3.1) has a purely combinatorial character. However, we do not know its straightforward proof and we have to make use of a sufficiently artificial auxiliary construction.

Let $\Lambda$ be an associative algebra with $n+m$ generators $\lambda_{1}, \ldots, \lambda_{n+m}$, defined by the following .relations: any (noncommutative) monomial of $\lambda_{1}, \ldots, \lambda_{n+m}$, having degree not less than two at least with respect to one variable, is equal to zero.

For each $k>0$ there is defined the right action of the group $\Sigma_{k}$ on the polynomials of degree $k$ of $\lambda_{1}, \ldots, \lambda_{n+m}$ by the rule

$$
\sigma \lambda_{i_{1}} \cdot \ldots \cdot \lambda_{i_{k}}=\lambda_{l_{\sigma(1)}} \cdot \ldots \cdot \lambda_{i_{\sigma(k)}} \forall \sigma \in \Theta_{-k} \cdot
$$

We have $(\sigma \circ v) \lambda_{i_{1}} \cdot \ldots \cdot \lambda_{i_{k}}=v\left(\sigma \lambda_{i_{1}}, \ldots \cdot \lambda_{i_{k}}\right)$.

Let $\eta=\left\{\eta_{1}, \eta_{2}, \ldots, \eta_{n}, \ldots\right\}$ be a sequence of monomials of the first degree of the algebra $\Lambda$, having only a finite number of nonzero elements. We set

$$
\Delta_{k}(\eta)=\sum_{i_{1}<\ldots<i_{k}} \eta_{i_{i}} \cdots \cdots \cdot \eta_{i_{k}}
$$

By the letter $\lambda$ we denote the standard sequence $\lambda=\left\{\lambda_{1}, \ldots, \lambda_{n+m}, 0,0, \ldots\right\}$; thus, $\lambda_{k}=0$ for $k>n+m$. We have, in particular, $\Delta_{n+m}(\lambda)=\lambda_{1} \cdot \cdot \lambda_{n+m}$.

It is easy to verify the following identity, valid for any sequence and any $\mathrm{k}, l \geq 0$ :

$$
\Delta_{k}(\eta) \Delta_{l}(\eta)=\left(1_{k} w l_{l}\right) \Delta_{k+l}(\eta)
$$

Indeed,

$$
\Delta_{k}(\eta) \Delta_{l}(\eta)=\sum_{\substack{l_{1}<\ldots<l_{k} \\ l_{k+1}<\cdots<i_{k+l}}} \eta_{l_{1}} \cdot \cdots \cdot \eta_{l_{k+l}}=\sum_{\sigma \in S_{k+l}(k) j_{l}<\cdots<j_{k+l}} \eta_{J_{\sigma(1)}} \cdot \ldots \cdot \eta_{j_{\sigma(k+l)}}
$$

(we have made use of the fact that $\eta_{i_{1}} \cdot \ldots \cdot \eta_{i_{k+1}}=0$ if $i_{j_{1}}=i_{j_{2}}$ for some $j_{1} \neq j_{2}$ ). 
Let $\mathrm{x}$ be an element from $\mathscr{Y}$ of the form (3.2). Then

$$
x_{n+m}=\sum_{i_{0}+\ldots+t_{k}=n+m} t_{0}^{l_{0}} a_{i_{0}}^{0} w t_{1}^{i_{1}} a_{i_{1}}^{1} w \ldots w t_{k}^{t_{k}} a_{i_{k}}^{k}
$$

From the element $\mathrm{x}$ we construct a special sequence $\eta=\eta(\mathrm{x})$, setting

$$
\begin{gathered}
\eta_{j(n+m)+l}=t_{j} \lambda a_{n+m}^{j}(l), j=0,1, \ldots, k, i=1, \ldots, n+m, \\
\eta_{l}=0 \text { for } l>(k+1)(n+m) .
\end{gathered}
$$

We have the identity

$$
\Delta_{l}(\eta)=x_{l} \Delta_{l}(\lambda) \vee l>0
$$

Indeed,

$$
\begin{gathered}
\Delta_{l}(\eta)=\sum_{\lambda_{1}<\ldots<j_{l}} \eta_{l_{1}} \cdot \ldots \cdot \eta_{j_{l}}= \\
=\sum_{l_{0}+\ldots+i_{k}=l}\left(t_{0}^{l_{0}} a_{i_{0}}^{0} \Delta_{i_{0}}(\lambda)\right)\left(t_{1}^{t_{1}} a_{i_{1}}^{l} \Delta_{i_{1}}(\lambda)\right) \ldots\left(t_{k}^{l_{k}} a_{i_{k}}^{k} \Delta_{l_{k}}(\lambda)\right)= \\
=\sum_{l_{0}+\ldots+i_{k}=t} t_{0}^{l_{0}} \cdot \ldots \cdot t_{k}^{l_{k}}\left(a_{i_{0}}^{0} \otimes \ldots \otimes a_{i_{k}}^{k}\right) \Delta_{l_{0}}(\lambda) \ldots \Delta_{i_{k}}(\lambda) .
\end{gathered}
$$

At the same time, according to (3.3), we have

$$
\begin{gathered}
\left(a_{i_{0}}^{0} \otimes \ldots \otimes a_{i_{k}}^{k}\right) \Delta_{l_{0}}(\lambda) \ldots \Delta_{l_{k}}(\lambda)= \\
=\left(a_{i_{0}}^{0} \otimes \ldots \otimes a_{i_{k}}^{k}\right)\left(\left(1_{i_{0}} w \ldots w 1_{i_{k}}\right) \Delta_{l}(\lambda)\right)= \\
=\left(\left(1_{i_{0}} w \ldots \omega 1_{i_{k}}\right)\left(a_{i_{0}}^{0} \otimes \ldots \otimes a_{l_{k}}^{k}\right)\right) \Delta_{l}(\lambda)=\left(a_{i_{0}}^{0} w \ldots w a_{i_{k}}^{k}\right) \Delta_{l}(\lambda) .
\end{gathered}
$$

Consequently,

$$
\Delta_{l}(\eta)=\sum_{l_{0}+\ldots+i_{k}-l} t_{0}^{l_{0}} \cdot \ldots \cdot t_{k}^{l}{ }^{k}\left(a_{i_{0}}^{0} w \ldots w a_{i_{k}}^{k}\right) \Delta_{l}(\lambda)=x_{l} \Delta_{l}(\lambda)
$$

Making use of the equalities (3.3), (3.4), it is already easy to prove equality (3.1). We apply the left-hand side of the identity to be proved to $\lambda_{1} \cdot \ldots \cdot \lambda_{n+m}=\Delta_{n+m}(\lambda)$ :

$$
\begin{gathered}
\left(x_{n+m^{\circ}}\left(1_{m} w 1_{n}\right)\right) \Delta_{n+m}(\lambda)=\left(1_{m} w 1_{n}\right)\left(x_{n+m} \Delta_{n+m}(\lambda)\right)= \\
=\left(1_{m} w 1_{n}\right) \Delta_{n+m}(\eta)=\Delta_{m}(\eta) \cdot \Delta_{n}(\eta)=\left(x_{m} \Delta_{m}(\lambda)\right)\left(x_{n} \Delta_{n}(\lambda)\right)= \\
=\left(x_{m} \otimes x_{n}\right) \Delta_{m}(\lambda) \Delta_{n}(\lambda)=\left(x_{m} \otimes x_{n}\right)\left(\left(1_{m} w 1_{n}\right) \Delta_{n+m}(\lambda)\right)= \\
=\left(\left(x_{m} \otimes x_{n}\right) \circ\left(1_{m} w 1_{n}\right)\right) \Delta_{n+m}(\lambda)=\left(\lambda_{m} w x_{n}\right) \Delta_{n+m}(\lambda) .
\end{gathered}
$$

Consequently, $x_{n+m^{\circ}}\left(I_{m} w 1_{n}\right)=x_{m} w x_{n}$.

2. A surprising consequence of the fundamental lemma is the fact that the symmetric product of two elements of a shuffle algebra is again an element of the shuffle algebra. Moreover, this lemma enables us to describe completely the table of the symmetric multiplication of the elements of an additive basis of a shuffle algebra.

PROPOSITION 9. For any $n, m>0$ and positive integers $i_{1}, \ldots, i_{n}, j_{1}, \ldots, j_{m}$ we have the equality

$$
\begin{aligned}
& \left(1_{l_{2}} w \ldots w 1_{l_{n}}\right)\left(l_{l_{1}} w \ldots w l_{l_{m}}\right)= \\
& =\sum_{K} 1_{k_{11}} w \ldots w 1_{k_{n 1}} w 1_{k_{11}} w \ldots w 1_{k_{22}} \mathrm{w} \ldots w 1_{k_{1 m}} \mathrm{w} \ldots w 1_{k_{n m^{\prime}}}
\end{aligned}
$$

where the summation is taken over all matrices

$$
K=\left(\begin{array}{ccc}
k_{11} & \ldots & k_{1 m} \\
\vdots & & \vdots \\
\dot{k}_{n 1} & \ldots & k_{n m}
\end{array}\right)
$$


satisfying the conditions

$$
\begin{aligned}
& \sum_{l=1}^{n} k_{l l}=j_{l}, \quad l=1, \ldots, m ; \\
& \sum_{j=1}^{m} k_{l j}=i_{l}, \quad l=1, \ldots, n .
\end{aligned}
$$

Proof. From the fundamental lemma there follows that

$$
\begin{aligned}
& \left(\exp \left(t_{1}\right) w \ldots w \exp \left(t_{n}\right)\right) \circ\left(1_{j}, w \ldots w I_{J_{m}}\right)= \\
& =\left(\left(\exp \left(t_{1}\right) w \ldots ш \overrightarrow{\exp }\left(t_{n}\right)\right)>1_{J_{1}}\right) w \ldots w\left(\left(\exp \left(t_{1}\right) ш \ldots \omega \exp \left(t_{n}\right)\right) \circ 1_{J_{m}}\right) \text {. }
\end{aligned}
$$

Making equal the coefficients of $t_{1}{ }_{1}{ }^{\prime} \cdot \ldots \cdot t_{n}{ }_{n}$ in the right- and left-hand sides, we obtain the desired equality.

Thus, $\forall \mathrm{n}>0$ the space $!_{\mathrm{n}}$ is an algebra relative to the symmetric multiplication " $\circ$ ", a subalgebra in the group algebra of the symmetric group $\Sigma_{n}$. The space $\ddot{i}_{n}$ with the symmetric multiplication will be denoted by $\dot{U}_{n}$, while the space $W_{+}=\bigoplus_{n=1}^{\infty} W_{n}$ - with the same multiplication will be denoted by the symbol $\dot{U}_{+}$. We note that $W_{n^{2}} L I I_{m}=0$ for $\mathrm{n} \neq \mathrm{m}$.

The circlets over $\ldots n$ and $\ldots+$ are used in order to distinguish the symmetric multiplication from the shuffle multiplication. Everywhere below (just as above) the symbol ${ }_{-}$without a circlet denotes the shuffle algebra, $\cdots+-$ i.e. is its maximal ideal, while $W_{+}^{k}=\underbrace{W_{+} \ldots \ldots W_{+}}_{\mathrm{k} \text { times }}$ is the $\mathrm{k}$-th power of the maximal ideal.

COROLLARY. The subspace $\cdots_{+}{ }^{\mathrm{k}}$ is a two-sided ideal in $\dot{H}_{+}$, i.e., $W_{+} \circ W_{+}^{k} \subset W_{+}^{k}, W_{+}^{k} W_{+} \subset W_{+}^{k}$.

This follows directly from Proposition 9.

We note that $\mathbf{1}_{\mathrm{n}}$ is the identity element in $\dot{L}_{\mathrm{n}}$, while the subspace $\Psi_{n} \cap W_{+}^{2}$ is an ideal of codimension 1 in $\dot{U}_{\mathrm{n}}$.

Definition. We denote by $\rho: \dot{W} \rightarrow \mathbf{R}$ the homomorphism of the algebra $\dot{W}$ into $\mathbf{R}$, defined in the following manner: if $\mathrm{x} \in \mathrm{-}_{\mathrm{n}}, \mathrm{X}=\mathrm{c} 1_{\mathrm{n}}+\mathrm{y}$, where $\mathrm{y} \in \mathrm{H}_{+}{ }^{2}$, then $\rho(\mathrm{x})=\mathrm{c}$.

We give the values of the homomorphism $\rho$ on the elements iaj, forming the natural additive basis of $\because$; here $a$ is a word in the alphabet $\{\alpha, \beta\}$.

PROPOSITION 10. Let $\operatorname{deg}_{\beta} a$ be the number of letters $\beta$ in the word $a$. Then $\rho(a)=(-1)^{\operatorname{deg}_{\beta} a}$.

Proof. The required equality follows directly from the definition of $\rho$ and the formula (2.1).

Remark. The functional $\rho$ on the algebra of the "monotonicity types of permutations" $\dot{U}_{n}$ plays a role similar to the role of the parity for individual permutations. We recall that by the letter $\beta$ we denote the places where the permutations of the given monotonicity type decrease (i.e., are in disarray!). On the other hand, $\rho$ does not coincide at all with the parity: for example, for the inversion $J_{n}=i \beta^{n-1} j$, having parity $(-1)^{n(n-1) / 2}$, we obtain $\rho\left(J_{n}\right)=(-1)^{n-1}$. The point is that at the computation of $\rho$ we do not take into account all the disarrays but only those that correspond to adjacent values of the argument.

The infinitesimal variant of the fundamental lemma is the following

PROPOSITION 11. For any $n>0, \vartheta \in V_{n}, x \in \mathbb{L}_{n}=0$ we have the equality $\vartheta \circ x=\rho(x) \vartheta$.

Proof. First of all, $\vartheta \circ 1_{n}=\vartheta$. Therefore, the assertion to be proved is equivalent to the relation $\vartheta \circ U_{+}^{2}=0$. We have $e^{\imath \bullet} \in \overline{\mathcal{Y}}$. $\vee \varepsilon \in R$, where the exponential series is considered relative to the shuffle multiplication:

$$
e^{e \theta}=1+\sum_{n=1}^{\infty} \frac{8^{n}}{n !} \underbrace{\vartheta \amalg \ldots . . . \vartheta}_{n \text { times }} .
$$

From the fundamental lemma there follows that $e^{\varepsilon \theta}\left(x_{1} \amalg x_{2}\right)=\left(e^{\varepsilon \vartheta_{0}} x_{1}\right) \amalg\left(e^{e \vartheta_{0}} x_{2}\right), v_{1}, x_{2} \in \Psi_{7}$. Differentiating this identity with respect to $\varepsilon$ at $\varepsilon=0$, we obtain

$$
\vartheta \circ\left(x_{1} ш x_{2}\right)=\left(\vartheta \circ x_{1}\right) \Psi\left(e^{0} \circ x_{2}\right)+\left(e^{0} \circ x_{2}\right) ш\left(\vartheta \circ x_{2}\right)=0 .
$$

We recall that the associative algebra $W$ is the universal enveloping algebra of the Lie algebra V. In particular, the subspaces $\mathrm{V}^{\mathrm{n}}$ form an increasing filtration in $\Psi_{+}$. 
THEOREM 3. 1) $V^{n}=\left\{x \in U \mid x_{\circ} U_{+}^{n+1}\right\}=0, \forall n>0$. 2) Let $\vartheta_{1}, \ldots, \vartheta_{\mathrm{n}} \in \mathrm{V}$ be homogeneous elements. For an

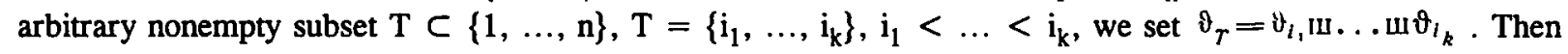

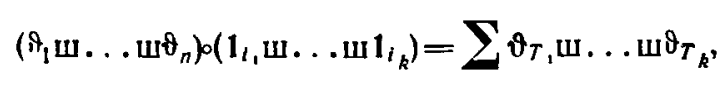

where the summation is taken over all partitions of $\{1, \ldots, \mathrm{n}\}$ into $\mathrm{k}$ subsets $\mathrm{T}_{\mathrm{j}}$ such that $\vartheta_{T_{j}} \in W_{i j}, j=1, \ldots, k$.

Proof. We start with statement 2). Equality (3.5) is a direct generalization of Proposition 11. The same can be said about the proof. For any $t_{1}, \ldots, t_{n} \mathbf{R}$ the element $e^{t_{1} \theta_{1}} w \ldots w e^{t_{n} \vartheta_{n}}$ lies in $\overline{\mathcal{P}}$. Consequently,

$$
\begin{aligned}
& \left(e^{t_{1} \theta_{1}, \mathrm{II}} \ldots \mathrm{w} e^{t^{\theta^{\theta}} n}\right)_{\circ}\left(1_{i_{1}} \mathrm{w} \ldots \mathrm{w} \mathbf{1}_{i_{k}}\right)=
\end{aligned}
$$

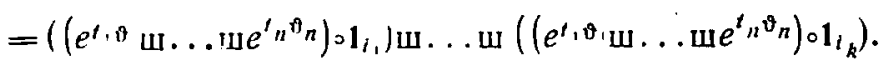

Making equal the coefficients of $t_{1} \cdot \ldots \cdot t_{n}$ in the right- and left-hand sides of the last equality, we obtain (3.5).

We proceed to the proof of statement 1). First of all, from (3.5) there follows that $V^{n} U I_{+}^{n+1}=0$. It remains to show that $x: U_{\perp}^{\prime \prime-1} \neq 0$. if $\mathrm{x} \notin \mathrm{V}^{n}$.

Let $\vartheta_{1}, \vartheta_{2}, \ldots$ be an additive basis of $\mathrm{V}$, consisting of homogeneous elements, linearly ordered in such a manner that the degrees of the elements do not decrease monotonically. From the Poincaré-Birkhoff-Witt theorem there follows that the

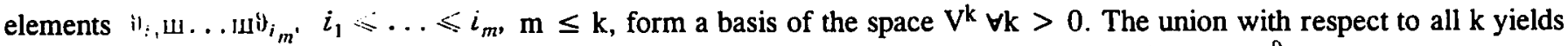
an additive basis of the space $\ldots$. For each $x \in \ldots$ by $x_{i_{1}} \ldots i_{m}$ we denote the coefficient of $v_{i}, w \ldots w i_{i_{m}}$ in the expansion of $\mathrm{x}$ with respect to this basis.

Let $x \in V^{k} \backslash V^{k-1}$; by the principal term of the expansion of $x$ with respect to the given basis we mean the element

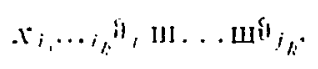

where $j_{1}, \ldots, j_{k}$ is lexicographically minimal among all sequences $i_{1}, \ldots, i_{k}$ such that $x_{i_{1}} \ldots i_{k} \neq 0$.

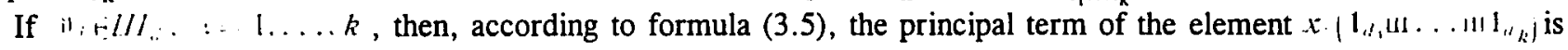
equal to

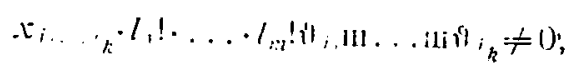

where $l_{1}, \ldots, l_{\mathrm{m}}$ are the lengths of the constancy intervals of the nondecreasing integer-valued function $i \cdots d_{i}, i=1 \ldots \ldots$

COROLLARY. The subspaces $V^{n}$ are two-sided ideals in $I I_{.}: V^{n} I I I_{-}=U_{-} \circ V^{n}=V^{\prime}$

3. Now we leave for a short time the bounds of the shuffle algebra $i$ in order to give some consequences of Theorem

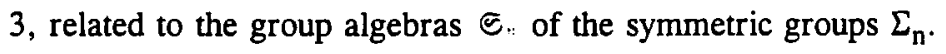

Let $\lambda_{1}, \ldots, \lambda_{n}$ be independent generators (alphabet) of a free associative algebra. Associating to each permutation $\sigma$ $\in \Sigma_{\mathrm{n}}$ the monomial $\lambda_{\sigma}=\lambda_{\sigma(1)} \cdot \ldots \cdot \lambda_{\sigma(\mathrm{n})}$, while to the linear combination $\mathrm{x}=\sum_{i} \mathrm{c}_{\mathrm{i}} \sigma_{\mathrm{i}}$ of permutations the polynomial $\lambda_{\mathrm{x}}$ $=\sum_{i} c_{i} \lambda_{\sigma_{i}}$, we identify the group algebra $\bigodot_{n}$ with the subspace in Ass $\left(\lambda_{1}, \ldots, \lambda_{n}\right)$, consisting of elements that are homogeneous of degree unity with respect to each variable. We set

$$
\operatorname{Lie}_{n}=\left\{x \in S_{n} \mid \lambda_{x} \in \operatorname{Lie}\left(\lambda_{1}, \ldots, \lambda_{n}\right)\right\}
$$

It is easy to see that $\mathrm{Lie}_{\mathrm{n}}$ is a left ideal in the group algebra $\odot$, since a permutation of the variables in the commutator polynomial in $\lambda_{1}, \ldots, \lambda_{n}$ leaves the polynomial a commutator.

THEOREM 4. For any $n>0$ we have the following relations:

1) $V_{n}=\mathrm{Lie}_{n} \cap W_{n}$ :

2) if $\vartheta \in \mathrm{V}_{\mathrm{n}}, \rho(\vartheta) \neq 0$, then $\sigma_{n} \diamond \vartheta=\mathrm{Lie}_{n}$.

Proof. Let

$$
\pi: \operatorname{Ass}\left(\lambda_{1}, \ldots, \lambda_{n}\right) \rightarrow \operatorname{Lie}\left(\lambda_{1}, \ldots, \lambda_{n}\right)
$$

be a linear mapping, acting according to the rule

$$
\pi\left(\lambda_{i_{1}} \cdot \ldots \cdot \lambda_{i_{k}}\right)=\frac{1}{k} \operatorname{ad} \lambda_{i_{1}} \ldots \operatorname{add} \lambda_{i_{k-1}} \lambda_{i_{k}}
$$


It is well known that $\pi$ is a projection of $\operatorname{Ass}\left(\lambda_{1}, \ldots, \lambda_{n}\right)$ onto $\operatorname{Lie}\left(\lambda_{1}, \ldots, \lambda_{n}\right)$ (see [4]). Now we note that $\pi\left(\lambda_{x}\right)=$ $(1 / n) \lambda_{x} \circ \bar{\omega}_{n} \forall x \in \mathcal{S}_{n} \quad$ (compare with Subsection 1.4). Consequently, $\bar{\omega}_{\mathrm{n}}$ generates the left ideal Lie $\mathrm{n}_{\mathrm{n}}$, i.e., $\mathcal{S}_{n}{ }^{2} \omega_{n}=\mathrm{Lie}_{n}$. We mention that $\rho\left(\omega_{n}\right)=n$ and we set $\pi_{n}=(1 / n) \bar{\omega}_{n}$. The element $\pi_{n} \in V_{n}$ is an idempotent by virtue of Proposition 9: $\pi_{\mathrm{n}} \circ \pi_{\mathrm{n}}=1$. Consequently,

$$
x \mathrm{FS}_{1,0 \pi_{n}}=\mathrm{Lie}_{n} \Leftrightarrow x=\pi_{n}=x .
$$

From Proposition 9 there follows that ${ }^{\circ} \circ \pi_{n}=9 \vee G V_{n}$, while from the Corollary to Theorem 3 we obtain that $x \circ \pi_{n} \in V_{n}$ $\forall x \in I_{n}$

Thus, assertion 1) of the theorem is proved.

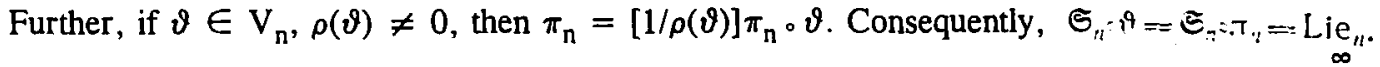

Let $\lambda s s(S)=\underset{n \rightarrow 0}{y_{A s s}}(S)$ be a free associative algebra with some set $S$ of generators, graded in the usual manner.

We define the right action $\theta_{n}$ of the algebra $\Xi_{n}$ on the space $\operatorname{Ass}_{\mathrm{n}}(\mathrm{S})$, by setting for all $x=\sum_{i} c_{l} \sigma_{i} \in \subseteq_{n}$

$$
\theta \because(. t): s_{1} \cdot \ldots \cdot s_{n} \mapsto \sum_{i} c_{i} s_{\sigma(1)} \cdot \ldots \cdot s_{\sigma(n)}
$$

where $s_{\mathrm{i}} \in \mathrm{S}, \mathrm{i}=1, \ldots, \mathrm{n}$, are arbitrary variables, not necessarily distinct .

COROLLARY. Let $\vartheta_{\mathrm{k}} \in \mathrm{V}_{\mathrm{k}}, \rho\left(\vartheta_{\mathrm{k}}\right)=1, \mathrm{k}=1,2, \ldots$. Then the mapping

$$
\bigoplus_{k=1}^{\infty} \theta_{k}\left(\vartheta_{k}\right): \operatorname{Ass}(S) \rightarrow \operatorname{Ass}(S)
$$

is a projection of $\operatorname{Ass}(\mathbf{S})$ onto $\operatorname{Lie}(\mathrm{S})$.

4. The results of the last two subsections contain the solution of the initial problems of this paper, formulated in Subsection 1.4: describe all the commutator representations of the $\mathrm{n}$-th variation and find the symmetries that transform an arbitrary representation of the $\mathrm{n}$-th variation again into some representation of the $\mathrm{n}$-th variation. In this case, as elucidated, the solution of the second problem includes, basically, the solution of the first problem.

Let $h_{t}$ be a nonstationary field. All the commutator representations of the $n$-th variation of the system (1.8) have the form

$$
h . \vartheta, \vartheta \in V_{n} \cap \rho^{-1}(1)
$$

By applying to $h_{\vartheta} \vartheta$ the symmetry $x \in \stackrel{\cdots}{-}$, we obtain the expression $h_{*}(x \circ \vartheta)$. Thus, the set of the symmetries from ${ }_{-n}$, which map the representations of the $n$-th variation again into representations of the $n$-th variation is ${ }_{n} \cap \rho^{-1}(1)$. In addition, for all $\vartheta_{1}, \vartheta_{2} \in \mathrm{V}_{\mathrm{n}} \cap \rho^{-1}(1)$ we have $\vartheta_{1} \circ \vartheta_{2}=\vartheta_{1}$.

Thus, by the application of symmetries to an arbitrary commutator representation, we obtain easily another commutator representation and there are no privileged commutator representations.

\section{EXTENSIONS}

1. So far we have investigated algebraic structures, connected with the asymptotic expansion of flows, generated by a fixed system of the form (1.8). In applications we encounter frequently the situation when on the right-hand sides of the considered systems there acts some group $\Gamma$ and it is necessary to compare and combine the asymptotic expansions of the systems obtained from the given one by the action of various elements of the group. In other words, it is necessary to extend the variational group in a special manner with the aid of the group $\Gamma$.

We obtain a simple and important example of the action of the group on the right-hand side by starting directly from a nonstationary system (1.8), without any additional structures. This is the action of the group of affine transformations of the time axis: $h_{t} h_{c_{1} t+c_{2}}, c_{1}, c_{2} \in \mathbf{R}, c_{1} \neq 0$. If the right-hand side depends on parameters, discrete or continuous, as this happens in control theory, then there acts the transformation group of the space of the parameters. For example, for the investigation of the interaction of $l$ systems with right-hand sides $h_{t}{ }^{1}, \ldots, h_{t}{ }^{l}$ one makes use of the symmetric group $\Gamma=\Sigma_{l}$, whose elements rearrange the fields $h_{\mathrm{i}}^{\mathrm{i}}$. 
2. We proceed to the description of the extension of the shuffle algebra with the aid of the group $\Gamma$. Let

$$
\Gamma^{n}=\left\{\gamma=\left(\gamma_{1}, \ldots, \gamma_{n}\right) \mid \gamma_{i} \in \Gamma, i=1, \ldots, n\right\}
$$

be the Cartesian product of $n$ copies of the group $\Gamma$,

$$
\gamma \cdot \gamma^{\prime}=\left(\gamma_{1} \cdot \gamma_{1}^{\prime}, \ldots, \gamma_{n} \cdot \gamma_{n}^{\prime}\right)
$$

If $\sigma \in \Sigma_{\mathrm{m}}$, then the correspondence

$$
\gamma=\left(\gamma_{1}, \ldots, \gamma_{n}\right) \rightarrow \gamma_{\sigma}=\left(\gamma_{\sigma(1)}, \ldots, \gamma_{\sigma(n)}\right)
$$

defines the right action of $\Sigma_{n}$ onto $\Gamma^{n}$.

Definition. By the symbol $\Sigma_{n}^{\Gamma}$ we denote the following semidirect product of $\Sigma_{n}$ by $\Gamma^{n}$ :

$$
\begin{aligned}
& \Sigma_{n}^{\Gamma}=\left\{(\gamma, \sigma) \mid \gamma \in \Gamma^{n}, \sigma \in \Sigma_{n}\right\}, \\
& \left(\gamma^{\prime}, \sigma^{\prime}\right) \circ(\gamma, \sigma)=\left(\gamma \cdot \gamma_{\sigma}^{\prime}, \sigma^{\prime} \circ \sigma\right) .
\end{aligned}
$$

The initial objects at the construction of the shuffle algebra have been the groups $\Sigma_{n}$; at the construction of the extended shuffle algebra the initial objects are the groups $\Sigma_{n}^{\Gamma}$, while multiplication in these groups plays the same role as the usual symmetric multiplication.

Assume that on some set $U$ there is defined a left action of the group $\Gamma$. The result of the application of the element $\gamma \in \Gamma$ to $u \in U$ will be denoted simply by $\gamma$ u. Let $\Phi: U^{n} \rightarrow \mathscr{D}$ be a mapping of $U^{n}$ into some set $\mathscr{D}$ (the selection of the letters is justified by the fact that, in our important applications, $U$ is the set of control parameters, while $\mathscr{D}$ is the algebra of differential operators on $\mathrm{M}$ ).

For $(\gamma, \sigma) \in \Sigma_{\mathrm{n}}^{\Gamma}$ we set

$$
(\gamma, \sigma) \Phi\left(u_{1}, \ldots, u_{n}\right)=\Phi\left(\gamma_{1} u_{\sigma(1)}, \ldots, \gamma_{n} u_{\sigma(n)}\right) .
$$

The multiplication (4.1) is defined in such a manner that the correspondence $\Phi \rightarrow(\gamma, \sigma) \Phi$ is a left action of the group $\Sigma$ n, i.e.,

$$
\left(\gamma^{\prime}, \sigma^{\prime}\right)((\gamma, \sigma) \Phi)=\left(\gamma^{\prime}, \sigma^{\prime}\right) \rho(\gamma, \sigma) \Phi
$$

We denote by $\varsigma_{n}^{\Gamma}$ the group algebra over $\mathbf{R}$ of the group $\Sigma_{n}^{\Gamma}, \varsigma_{0}^{\Gamma}=\mathbf{R}$, and $\mathcal{S}^{\Gamma}={\underset{n}{\oplus} 0}_{n}^{\infty} \mathcal{S}_{n}^{\Gamma}$.

We define the shuffle product of arbitrary $(\gamma, \sigma) \in \Sigma^{\mathrm{n}}$ and $\left(\gamma^{\prime}, \sigma^{\prime}\right) \in \Sigma_{\mathrm{m}}^{\Gamma}$ by the formula

$$
(\gamma, \sigma) \omega\left(\gamma^{\prime}, \sigma^{\prime}\right)=\left(\left(\gamma, \gamma^{\prime}\right), \sigma w \sigma^{\prime}\right) \in \Theta_{n+m}^{\Gamma}
$$

where $\left(\gamma, \gamma^{\prime}\right)=\left(\gamma_{1}, \ldots, \gamma_{n}, \gamma_{1}^{\prime}, \ldots, \gamma_{m}^{\prime}\right) \in \Gamma^{n+m}$.

The shuffle product is extended by linearity for any pair of elements of $\mathcal{S}^{\Gamma}$ and defines in $\mathcal{S}^{\Gamma}$ a structure of an associative graded algebra.

Let $U \subset \mathbf{R}^{\mathbf{N}}$, let $\mathscr{D}$ be a Fréchet space, assume that the mappings $u \rightarrow \gamma \mathrm{u}, \mathbf{u} \in \mathrm{U}$, are continuous $\forall \gamma \in \Gamma$, the mapping $\Phi: U^{n} \rightarrow \mathscr{D}$ is also continuous, and $u(\cdot):[0,1] \rightarrow U$ is a measurable bounded vector-valued function. It is easy to verify the identity

$$
\begin{gathered}
\left.\int_{\Delta^{k} \times \Delta^{n-k}} \ldots \int_{\nu_{1}} \Phi\left(\gamma_{1} u\left(t_{1}\right), \ldots, \gamma_{r} u\left(t_{k}\right), \gamma_{1}^{\prime} u\left(t_{k+1}\right), \ldots, \gamma_{n-k}^{\prime} u\left(t_{n}\right)\right)\right) d t_{1} \ldots d t_{n}= \\
\quad=\int_{\Delta^{n}} \ldots \int\left(\left(\gamma, 1_{k}\right) \omega\left(\gamma^{\prime}, 1_{n-k}\right)\right) \Phi\left(u\left(t_{1}\right), \ldots, u\left(t_{n}\right)\right) d t_{1} \ldots d t_{n}
\end{gathered}
$$

for any

$$
k=0,1, \ldots, n, \quad \gamma=\left(\gamma_{1}, \ldots, \gamma_{n}\right) \in \Gamma^{k}, \quad \gamma^{\prime}=\left(\gamma_{1}^{\prime}, \ldots, \gamma_{n-k}^{\prime}\right) \in \Gamma^{n-k}
$$

Let $u \rightarrow h(u)$ be some continuous mapping of $U$ into $\operatorname{Vect}(M)$. We set $H^{k}\left(u_{1}, \ldots, u_{k}\right)=h\left(u_{1}\right) \circ \ldots \circ h\left(u_{k}\right)$. We obtain an important special case of the identity (4.2) by taking for $\Phi$ the mapping

$$
\left(u_{1}, \ldots, u_{n}\right) \mapsto H^{k}\left(u_{\sigma(1)}, \ldots, u_{\sigma(k)}\right) H^{n-k}\left(u_{\sigma^{\prime}}(1), \ldots, u_{\sigma^{\prime}(n-k)}\right)
$$


where $\sigma \in \Sigma_{k}, \sigma^{\prime} \in \in_{n-k}$.

In this case $(4.2)$ reduces to the equality

$$
\begin{gathered}
\int \ldots \int(\gamma, \sigma) H^{k}\left(u\left(t_{1}\right), \ldots, u\left(t_{k}\right)\right) d t_{1} \ldots d t_{k}{ }^{\circ} \\
\quad \int_{\Delta^{k}} \ldots \int_{\Delta^{n-k}}\left(\gamma^{\prime}, \sigma^{\prime}\right) H^{n-k}\left(u\left(t_{1}\right), \ldots, u\left(t_{n-k}\right)\right) d t_{1} \ldots d t_{n-k}= \\
=\int \underset{\Delta^{n}}{\ldots}\left((\gamma, \sigma) w\left(\gamma^{\prime}, \sigma^{\prime}\right)\right) H^{n}\left(u\left(t_{1}\right), \ldots, u\left(t_{n}\right)\right) d t_{1} \ldots d t_{n} .
\end{gathered}
$$

Thus, the mapping

$$
\begin{gathered}
(\gamma, \sigma) \mapsto \int_{\Delta^{k}} \ldots \int(\gamma, \sigma) H^{k}\left(u\left(t_{1}\right), \ldots, u\left(t_{n}\right)\right) d t_{1} \ldots d t_{n}, \\
(\gamma, \sigma) \in \Sigma_{k}^{\Gamma}, \quad k=1,2, \ldots
\end{gathered}
$$

defines a homomorphism of the algebra $\varsigma^{\Gamma}$ into the algebra of differential operators on $M$.

3. The subgroup in Diff(M), generated by the diffeomorphisms

$$
\overrightarrow{\exp } \int_{0}^{1} \varepsilon h(\gamma u(t)) d t, \quad \varepsilon \in \mathrm{R}, \quad \gamma \in \Gamma,
$$

is called the $\Gamma$-extension of the variational group of the system

$$
\dot{x}=x \circ h(u(t)) \text {. }
$$

Correspondingly, by the $\Gamma$-extension of the Lie algebra of variations of the system (4.4) we mean the Lie subalgebra in Vect(M), generated by the Lie algebras of variations of the systems

$$
\dot{x}=x \circ h(\gamma u(t)), \gamma \in \Gamma \text {. }
$$

We define the abstract analogues of these entities by following the same scheme as in Sec. 2. First of all let $\overline{\mathfrak{S}}^{\mathrm{r}}$ be the completion of the graded algebra $\mathcal{S}^{\Gamma}=\bigoplus_{n=0}^{\infty} \mathfrak{S}_{n}^{\Gamma}$ in the standard topology of the convergence of the homogeneous components.

If $\gamma \in \Gamma$, then we denote

$$
\begin{aligned}
&(\gamma)_{n}\left.=(\underbrace{\gamma, \ldots, \gamma}_{n \text { pas }}), 1_{n}\right), \quad(\gamma)_{n} \in \Sigma_{n}^{\Gamma}, \quad(\gamma)_{0}=1 \in R ; \\
& \overrightarrow{\exp }(\varepsilon, \gamma)=\sum_{n=0}^{\infty} \varepsilon^{n}(\gamma)_{n}, \quad \overrightarrow{\exp }(\varepsilon, \gamma) \in \overline{\mathbb{S}}^{\Gamma} ; \\
& \overleftarrow{(i)}(\varepsilon, \gamma)=\sum_{n=1}^{\infty} \varepsilon^{n-1} \overleftarrow{\omega}_{n}(\gamma)=\frac{\partial}{\partial \varepsilon}(\overrightarrow{\exp }(\varepsilon, \gamma)) w(\exp (\varepsilon, \gamma))^{-1} .
\end{aligned}
$$

Definition. The subgroup $\mathscr{V}_{\Gamma}$ in the multiplicative semigroup of the algebra $\mathcal{S}_{1} \Gamma$, generated by the elements $\overrightarrow{\exp }(\varepsilon$, $\gamma), \varepsilon \in \mathbf{R}, \gamma \in \Gamma$, is called the $\Gamma$-extension of the abstract variational group.

The Lie subalgebra $V^{\Gamma}$ in $[\odot r]$, generated by the elements $\bar{\omega}_{n}(\gamma), n=1,2, \ldots, \gamma \in \Gamma$, is called the $\Gamma$-extension of the abstract Lie algebra of variations.

The $\Gamma$-extension of the abstract Lie algebra of variations is contained in the (associative) subalgebra of the algebra $\mathcal{S} \Gamma$, generated by the elements $(\gamma)_{n}, \gamma \in \Gamma, \mathrm{n}=0,1, \ldots$, while the $\Gamma$-extension of the abstract variational group lies in the closure of this subalgebra in $\overline{\bar{\sigma}} \mathbf{r}$.

Definition. The (associative) subalgebra in $\sigma^{r}$, generated by the elements $(\gamma)_{n}, \gamma \in \Gamma, n=0,1, \ldots$, is called the $\Gamma$-extension of the shuffle algebra and is denoted by the symbol $\amalg^{\Gamma}$, while its closure in $\mathfrak{S}^{\Gamma}$ is denoted by the symbol $\amalg^{\Gamma}$.

Clearly, $W^{\Gamma}$ is a graded subalgebra in $\mathrm{S}^{\Gamma}, W^{\Gamma}=\overbrace{n=0}^{\infty} W_{n}^{\Gamma}$, where $W_{n}^{\Gamma}=W^{\Gamma} \cap \mathrm{S}_{n}^{\Gamma}$. 


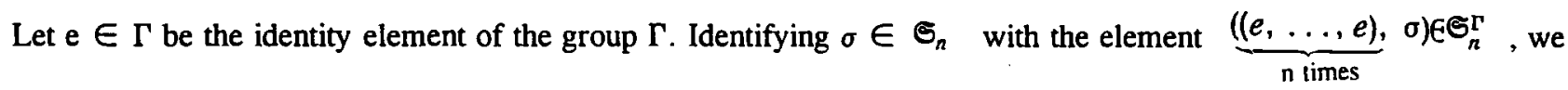

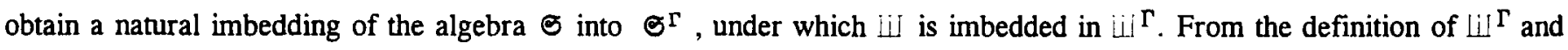
from the fact that $\mathrm{i}$ is a free algebra with generators $1_{n}, n=1,2, \ldots$ (see the corollary to Proposition 3 ) there follows that ${ }_{-} \Gamma$ is a free associative algebra with generators $(\gamma)_{n}, \gamma \in \Gamma, n=1,2, \ldots$. A simple generalization of Theorem 1 is Theorem 1 is

THEOREM $1^{\Gamma}$. The $\Gamma$-extension $V^{\Gamma}$ of the abstract Lie algebra of variations is a free Lie algebra with generators $\leftarrow \omega_{n}(\gamma) \in W_{n}$ r.

4. The extensions of the variational group and of the shuffle algebra, described briefly in the previous subsection, would be of little worth if the fundamental lemma, connecting the symmetric multiplication " $\circ$ " with the shuffle multiplication "sh?", would not extend to them.

As in Sec. 3, we extend the symmetric multiplication (initially defined separately on each of the homogeneous components $\mathfrak{S}_{n}^{\Gamma}$ ) to all of $\mathfrak{S}^{\Gamma}$, setting $a \circ \mathrm{b}=0$ for $a \in \mathfrak{S}_{n}^{\Gamma}, b \in \mathfrak{G}_{m}^{\Gamma}, n \neq m$.

The Fundamental Lemma for $\Gamma$-Extensions. For any $x \in \mathscr{V}^{\Gamma}$ and any $b^{\prime} \in \mathcal{S}^{\Gamma}$ we have the equality

$$
x \circ(a w b)=(x \circ a) w(x \circ b) .
$$

The proof follows the same scheme as the proof the fundamental lemma in Sec. 3 if we generalize in an appropriate manner the construction of the algebra $\Lambda$, used there. Namely, let $\Lambda^{\Gamma}$ be the associative algebra with generators $\left(\gamma, \lambda_{\mathfrak{j}}\right)$,

$$
\gamma \in \Gamma, i=1, \ldots, n+m \text {, }
$$

defined by the following relations: an arbitrary monomial $\left(\gamma_{1}, \lambda_{i_{1}}\right) \cdot \ldots \cdot\left(\gamma_{k}, \lambda_{i_{k}}\right)$, in which $i_{j}=i_{j^{\prime}}$ for some $j \neq j^{\prime}$, is equal to zero.

Instead of the right action of the group $\Sigma_{\mathrm{k}}$ on polynomials of degree $\mathrm{k}$ from the algebra $\Lambda$, used in Sec. 3, one has to consider the right action of the group $\Sigma_{\mathrm{k}}^{\Gamma}$ on polynomials of degree $\mathrm{k}$ from the algebra $\Lambda^{\Gamma}$, defined in the following manner:

$$
\begin{aligned}
(\gamma, \sigma):\left(\gamma_{1}, \lambda_{i_{1}}\right) \cdot \ldots \cdot\left(\gamma_{k}^{\prime}, \lambda_{i_{k}}\right) \mapsto\left(\gamma_{1} \cdot \gamma_{\sigma(1)}^{\prime}, \lambda_{i_{\sigma(1)}}\right) \ldots \cdot\left(\gamma_{k} \cdot \gamma_{\sigma(k)}^{\prime}, \lambda_{\left.i_{\sigma(k)}\right)}\right), \\
\forall \gamma=\left(\gamma_{1}, \ldots, \gamma_{k}\right) \in \Gamma^{k}, \sigma \in \Sigma_{k} .
\end{aligned}
$$

The remaining arguments differ very little from the proof of the fundamental lemma in Sec. 3 and we omit them.

Just as in the case of the usual shuffle algebra, the fundamental lemma enables us to describe completely the "table of the symmetric multiplication" in the $\Gamma$-extension of the shuffle algebra.

PROPOSITION 12. For any $n, m>0$, elements $\gamma_{1}, \ldots, \gamma_{n}, \gamma_{1}{ }^{\prime}, \ldots, \gamma_{m}{ }^{\prime} \in \Gamma$, and also positive numbers $i_{i}, \ldots$, $i_{n}, j_{1}, \ldots, j_{m}$ we have the equality

$$
\begin{aligned}
& \left(\left(\gamma_{1}\right)_{l}, \Psi \ldots ш\left(\gamma_{n}\right) l_{n}\right) \circ\left(\left(\gamma_{1}^{\prime}\right)_{j} ш \ldots \Psi\left(\gamma_{m}^{\prime}\right) j_{m}\right)= \\
& =\sum_{K}\left(\gamma_{1} \cdot \gamma_{1}\right)_{k_{1}} \omega \ldots \omega\left(\gamma_{1}^{\prime} \cdot \gamma_{n}\right)_{k_{n 1}} ш\left(\gamma_{2}^{\prime} \cdot \gamma_{1}\right)_{k_{1},} \text { ш. .ш }\left(\gamma_{2}^{\prime} \cdot \gamma_{n}\right)_{k_{n 2}} \Psi \ldots \\
& \ldots \omega\left(\gamma_{m}^{\prime} \cdot \gamma_{t}\right)_{k_{1} m} \omega \ldots ш\left(\gamma_{m}^{\prime} \cdot \gamma_{n}\right)_{n m},
\end{aligned}
$$

where the summation is taken over all matrices

$$
K=\left(\begin{array}{ccc}
k_{11} & \ldots & k_{1 m} \\
\vdots & \vdots \\
k_{n 1} & \ldots & k_{n m}
\end{array}\right)
$$

satisfying the conditions

$$
\sum_{i=1}^{n} k_{i l}=j_{l}, l=1, \ldots, m ; \quad \sum_{j=1}^{m} k_{l j}=i_{l}, l=1, \ldots, n .
$$

This statement is proved in exactly the same way as Proposition 7 in Sec. 3.

From it there follows that for each $n>0$ the space $\varpi_{n} \Gamma$ is an algebra relative to the symmetric multiplication " $\circ$ ", a subalgebra in the group algebra of the group $\Sigma_{n}^{\Gamma}$. We denote this "symmetric" algebra $\Psi_{n} \Gamma$ by $\dot{W}_{n} \Gamma$ in order to distinguish it from the shuffle algebra. 
Let $U_{+}^{\Gamma}=\oplus_{n=1}^{\infty} W_{n}^{\Gamma}$; from Proposition 12 there follows that $\left.W_{+}^{\Gamma} \Psi_{+} U_{+}^{\Gamma}\right) \cap U_{n}^{\Gamma}$ is a two-sided ideal in $\dot{U}_{n}^{\Gamma}$. Any element $\mathrm{x} \in \dot{U}_{\mathrm{n}}^{\Gamma}$ has the form

$$
\lambda=\sum_{i} c_{i}\left(\gamma_{i}\right)_{n}+y, \text { where } y \in W_{+}^{\Gamma} \omega U_{+}^{\Gamma}, c_{i} \in \mathbf{R}, \gamma_{i} \in \Gamma
$$

The mapping $\rho^{\Gamma}: x \mapsto \sum_{i} c_{l}\left(\gamma_{i}\right)_{n}$ is an antihomomorphism of $\dot{U}_{\mathrm{n}}{ }^{\mathrm{r}}$ into the group algebra of the group $\Gamma$.

The following statement, an infinitesimal variant of the fundamental lemma, is proved in the same way as Proposition 11.

PROPOSITION 13. For any $n>0, \vartheta \in V^{\Gamma} \cap W_{n}^{r}, x \in W_{n}^{r}$ we have the equality

$$
\vartheta \circ x=\vartheta \circ\left(\rho^{\Gamma}(x)\right)_{\kappa} .
$$

Concluding this section and at the same time also the paper, we note that the investigation of the $\Gamma$-extensions of a shuffle algebra and of an abstract Lie algebra of variations is only outlined here. More detailed information about these objects and their applications will be presented in subsequent publications.

\section{REFERENCES}

1. A. A. Agrachev and R. V. Gamkrelidze, "Exponential representation of flows and chronological calculus, "Mat. Sb., 107 (149), No. 4, 467-532 (1978).

2. R. V. Gamkrelidze, A. A. Agrachev, and S. A. Vakhrameev, "Ordinary differential equations on vector bundles, and chronological calculus," Itogi Nauki i Tekhniki, Ser. Sovr. Probl. Mat. Noveish. Dostizh., 35, 3-107 (1989).

3. A. A. Agrachev, R. V. Gamkrelidze, and A. V. Sarychev, "Local invariants of smooth control systems," Acta Appl. Math., 14, No. 3, 191-237 (1989).

4. N. Bourbaki, Lie Groups and Lie Algebras, Addison-Wesley, Reading (1975).

5. P. E. Crouch and F. Lamnabhi-Lagarrigue, "Algebraic and multiple integral identities," Acta Appl. Math., 15, No. 3, 235-274 (1989).

6. M. Fliess, "Fonctionnelles causales non linéaires et indéterminées non commutatives," Bull. Soc. Math. France, 109, No. 1, 3-40 (1981). 